\title{
Experimental and theoretical study of swept-wing boundary-layer instabilities. Three-dimensional Tollmien-Schlichting instability
}

Cite as: Phys. Fluids 31, 114104 (2019); https://doi.org/10.1063/1.5125812

Submitted: 28 August 2019 . Accepted: 23 October 2019 . Published Online: 18 November 2019

V. I. Borodulin (D, A. V. Ivanov, Y. S. Kachanov (D), D. A. Mischenko, R. Örlü (D), A. Hanifi, and S. Hein

\section{ARTICLES YOU MAY BE INTERESTED IN}

Three-dimensional simulation of tracer transport dynamics in formations with highpermeability channels or fractures: Estimation of oil saturation

Physics of Fluids 31, 113604 (2019); https://doi.org/10.1063/1.5120415

Vortex shedding patterns in flow past a streamwise oscillating square cylinder at low Reynolds number using dynamic meshing

Physics of Fluids 31, 113605 (2019); https://doi.org/10.1063/1.5123347

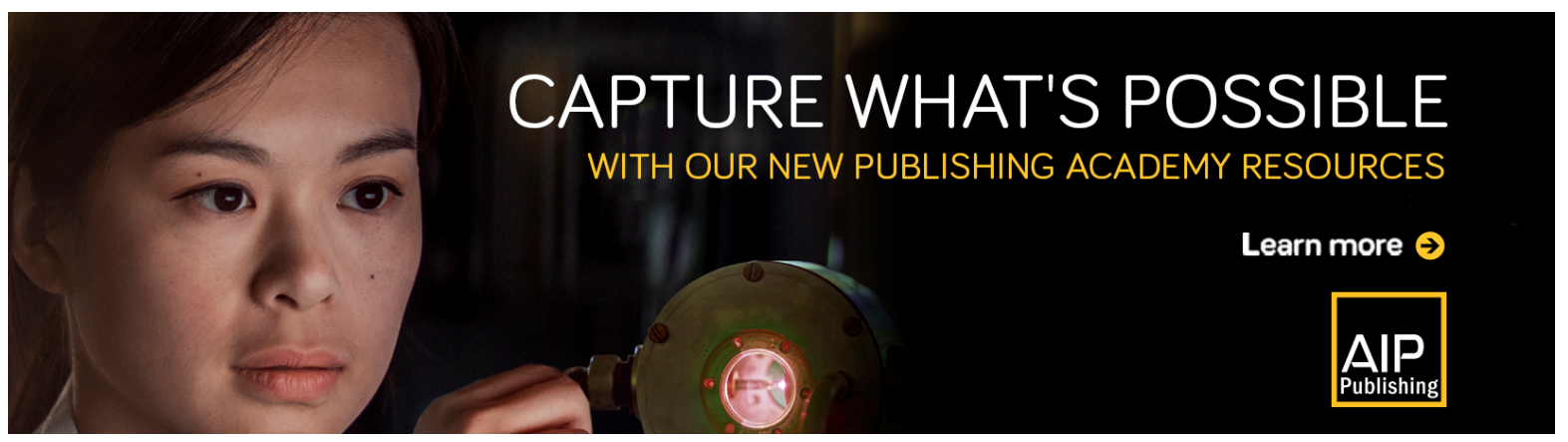




\title{
Experimental and theoretical study of swept-wing boundary-layer instabilities. Three-dimensional Tollmien-Schlichting instability
}

\author{
Cite as: Phys. Fluids 31, 114104 (2019); doi: 10.1063/1.5125812 \\ Submitted: 28 August 2019 - Accepted: 23 October 2019 • \\ Published Online: 18 November 2019
}

\author{
V. I. Borodulin, \\ A. V. Ivanov, Y. S. Kachanov, \\ D. A. Mischenko, ${ }^{1}$ R. Örlü, ${ }^{2}$ \\ A. Hanifi, ${ }^{2}$ and S. Hein ${ }^{3}$

\begin{abstract}
AFFILIATIONS
${ }^{1}$ Khristianovich Institute of Theoretical and Applied Mechanics, Novosibirsk 630090, Russian Federation

${ }^{2}$ KTH Royal Institute of Technology, Linné FLOW Centre, Department of Mechanics, SE-100 44 Stockholm, Sweden

${ }^{3}$ Institute of Aerodynamics and Flow Technology, DLR, 37073 Göttingen, Germany
\end{abstract}

\begin{abstract}
Extensive combined experimental and theoretical investigations of the linear evolution of three-dimensional (3D) Tollmien-Schlichting (TS) instability modes of 3D boundary layers developing on a swept airfoil section have been carried out. The flow under consideration is the boundary layer over an airfoil at $35^{\circ}$ sweep and an angle of attack of $+1.5^{\circ}$. At these conditions, TS instability is found to be the predominant one. Perturbations with different frequencies and spanwise wavenumbers are generated in a controlled way using a row of elastic membranes. All experimental results are deeply processed and compared with results of calculations based on theoretical approaches. Very good quantitative agreement of all measured and calculated stability characteristics of swept-wing boundary layers is achieved.
\end{abstract}

Published under license by AIP Publishing. https://doi.org/10.1063/1.5125812

\section{INTRODUCTION}

Prediction and control of laminar-turbulent transition, due to its significant impact on drag, is of great importance for aeronautical applications. Despite the long history of research on this subject, an accurate prediction of transition, especially in three-dimensional flows, still is not possible. This is due to the complexity of different stages of the transition process and the wide range of instability modes and their source of generation.

A rather detailed introduction into the problem under study is made in the first part of this work. ${ }^{1}$ Therefore, we present below only the most important points of this introduction related to the essence of the problem.

Among all types of instabilities possible in swept-wing boundary layers, there are two most important kinds of instability modes, which are able to lead to the laminar-turbulent transition on swept wings: the cross-flow (CF) modes and the Tollmien-Schlichting (TS) modes. The role of these modes in the transition process depends on certain flow parameters as the sweep angle and the chordwise pressure gradient. For instance, the CF-instability is the dominant mechanism in cases of the large sweep angle and negative (favorable) streamwise pressure gradient, while at small sweep angles or/and very weak favorable and positive (adverse) chordwise pressure gradients, the TS-instability waves are the dominant ones. Although the instability of swept-wing boundary layers has been studied intensively for many years (see, e.g., Refs. 2 and 3 for review), the detailed quantitative agreement between all measured and calculated linear stability characteristics has not been obtained previously for flows over real swept airfoils. This is especially true for nonstationary CFinstability modes. A breakthrough in the case of the nonstationary CF-instability has been achieved only recently in Ref. 1 . This kind of quantitative comparison for the 3D TS-instability characteristics of swept-wing boundary layers does not exist. This is mainly due to the extreme complexity of accurate detailed flow measurement over such geometries.

In general, there are several mechanisms of instability in the swept-wing boundary layers, each having particular characteristics as well as sources of their excitation. The most significant of these instability mechanisms are (a) the attachment-line flow instability, ${ }^{4}$ (b) the cross-flow instability prevailing in regions with large pressure gradients, especially favorable ones (see, e.g., Refs. 5 and 6), (c) the Tollmien-Schlichting instability that is most significant in 
regions of nearly zero and adverse pressure gradients (see, e.g., Ref. 7), and (d) the instability of separated shear layers that is important in the presence of laminar separation zones (see, e.g., Ref. 8).

The linear instability characteristics of 2D boundary layers with respect to 3D TS-waves have been investigated rather intensively. Detailed quantitative combined experimental and theoretical investigations of the 3D TS-instability of the Blasius boundary layer were performed in Refs. 9-15. Similar studies of the 3D TS-instability of an adverse-pressure-gradient boundary layer (with Hartree parameter $\beta_{H}=-0.115$ ) were carried out in Refs. 16 and 17. In all these experiments, the method of decomposition of wavetrains (harmonic in time and localized in the spanwise direction) into oblique TS-modes was developed and applied. It was shown that even classical locally parallel stability theory is able to describe rather accurately the increments, wall-normal profiles, and other characteristics of the 3D TS-waves experimentally observed in 2D boundary layers although the effects of the base-flow nonparallelism can be significant in some cases and can influence, in particular, the shape and location of the neutral stability curve (see also Ref. 18).

However, to the authors' knowledge, there are no works reported on investigation of 3D TS-instability in three-dimensional boundary layers.

It is clear at present that the location of the TS-dominated boundary-layer transition on swept wings depends on four main aspects: (i) spectra of the most dangerous flow and surface perturbations, (ii) boundary-layer receptivity characteristics, (iii) TS-instability characteristics, and (iv) leading mechanisms of nonlinear disturbance interactions. The present paper (the second part of the study) is devoted to a detailed quantitative experimental and theoretical investigation of aspect (iii), i.e., to the TS-instability characteristics of a 3D boundary layer developing on an airfoil section simulating an infinite-span swept wing.

This work was a part of the European research project "RECEPT" on the receptivity of three-dimensional boundary layers to surface roughness and freestream vortices. The development of the appropriate experimental procedure for the receptivity and stability measurements was one of the main goals of the present study. The first part of the work on excitation of crossflow perturbation has been presented in Ref. 1 .

\section{EXPERIMENTAL PROCEDURE AND THEORETICAL APPROACHES}

\section{A. Experimental setup}

The measurements were performed in the Minimum Turbulence Level (MTL) wind tunnel of the Royal Institute of Technology (Stockholm) at a freestream speed of about $11 \mathrm{~m} / \mathrm{s}$. The model is a swept wing $\left(35^{\circ}\right.$ sweep angle) with a constant cross section (modified NACA 67 1-215 airfoil) and a chord length of $c=800 \mathrm{~mm}$ (in the direction perpendicular to the leading edge). The model is the same as used in Ref. 1 but mounted at a $+1.5^{\circ}$ angle of attack and equipped with contoured sidewalls adjusted for this specific angle. The sidewalls were designed using the approach described in Refs. 19-21 to provide the required spanwise uniformity of the base flow. A general view on the experimental model and the sidewalls is given in Fig. 1(a).

\section{B. Coordinate system definition}

Coordinate systems used in the present paper are the following. The $x$-axis is directed parallel to the incident flow velocity vector $C_{0}$, along the test section centerline. The $z$-axis is normal to the $x$-axis [Fig. 1(a)]. The point $(x, z)=(0,0)$ corresponds to the airfoil leading edge at the middle section. The associated velocity components are $U$ and $W$. Another coordinate system used is $\left(x^{\prime}, z^{\prime}\right)$, which is obtained by two subsequent rotations of the $(x, z)$ system. First, both the $x$-axis and $z$-axis were turned by $35^{\circ}$ (sweep angle) around the axis normal to the $(x, z)$-plane passing through the point $(0,0)$. The next turn was made around the leading edge by $+1.5^{\circ}$ (angle of attack). As a result, the $z^{\prime}$-axis coincides with the leading edge and the chord line lies on the $x^{\prime}$-axis. The value $x^{\prime} / c=1$ corresponds to the trailing edge of the experimental model $(c=800 \mathrm{~mm})$. The associated velocity components in $x^{\prime}$ and $z^{\prime}$ directions are $U^{\prime}$ and $W^{\prime}$, respectively. One more important streamwise coordinates is the curvilinear $x_{s}^{\prime}$. It starts at the model leading edge and goes in the chordwise direction (similar to the $x^{\prime}$-axis) but along the model surface. The $y$-coordinate is locally normal to the model surface, and its origin $y=0$ lies always on the airfoil surface.

\section{Flow measurements}

To experimentally obtain the streamwise and spanwise potential-flow velocity components, the standard double-wire
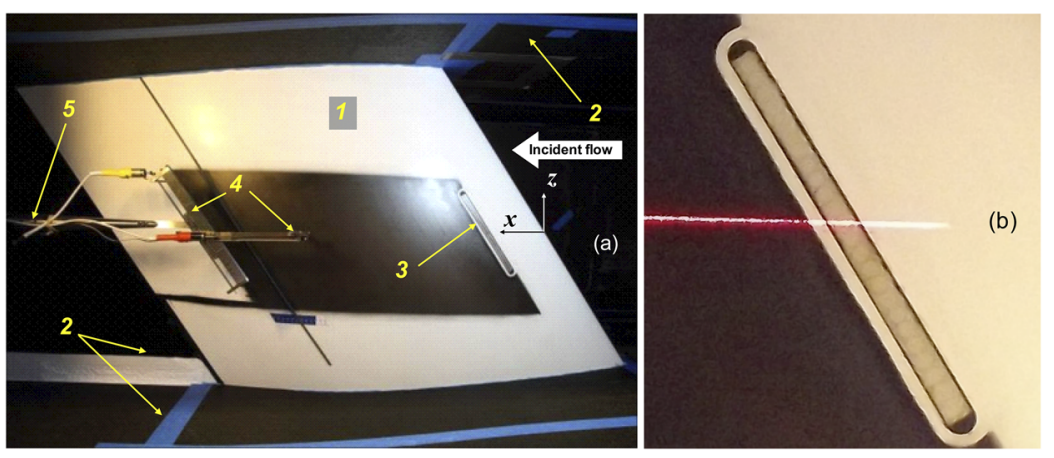

FIG. 1. Experimental model of a $35^{\circ}$ swept wing installed in the wind-tunnel test section at $a+1.5^{\circ}$ angle of attack (a) and source of controlled nonstationary surface nonuniformities with a spanwise row of latex membranes having a spanwise spacing of $\lambda_{z}^{\prime}=8 \mathrm{~mm}$ (b). 1: airfoil section, 2: contoured sidewalls, 3 : surface disturbance source, 4 : traverse device "Komarik," and 5: sting of standard wind-tunnel traverse. 
hot-wire measurements were performed in the potential flow at some distance from the airfoil surface (see Ref. 20). These data were used for accurate matching of the numerical flow characteristics with the experimental ones. The measurements also provided information about the accuracy of the infinite-sweep condition at large distances from the surface, as well as about blockage effects produced by the main MTL traverse. Additional two-component measurements were performed in the vicinity of the boundary-layer edge by means of a "wake-wire technique" combined with measurements by singlewire probes. This technique is described in Ref. 22. Subsequent processing of the wake-wire and single hot-wire measurements gave us potential-flow streamlines in a rather extended streamwise range of about $400 \mathrm{~mm}$ covering the whole region of interest. These types of data enabled also performing separation of the velocity components and finding, in particular, the spanwise components of the potential flow velocity vectors. The results of such measurements are presented in Sec. III A. Some details related to the experimental setup can be also found in Refs. 19-21.

The detailed measurements inside the boundary layer were performed using a boundary-layer type, single-wire hot-wire anemometry probe attached to the "Komarik" high-precision traversing system. The hot-wire itself is a Tungsten wire of $1 \mathrm{~mm}$ length and a nominal diameter of $5 \mu \mathrm{m}$. The hot wire was installed along the $z$-coordinate and measured with high accuracy the $U_{s}$-velocity component in all main measurements performed inside the boundary layer and near its edge. This component is parallel to the wall, but at the present experimental conditions, it coincides practically with the $U$-velocity component. Estimates, obtained based on a mathematical model of displacement of the traverse and the hot wire, have shown that slight inclinations of the hot wire during displacement of the traverse along the airfoil surface do not lead to any significant deviations of the measured velocity from the $U$-velocity component. This is true both for the mean velocity vector and for the vector of velocity fluctuations. The calibration of the hot-wire was performed in situ in the potential flow, approximately $30 \mathrm{~mm}$ away from the model surface, against a Pitot-Prandtl tube (inserted into the test section for calibration purposes only), connected to a FC0510 micromanometer, from which also the ambient temperature and pressure were obtained.

For accurate positioning of the hot-wire probe and the PitotPrandtl tube during calibration and measurements, a laser sheet was used. The sheet lay in the plane, which was parallel to the incident flow direction and perpendicular to the $z$-axis. The hot-wire anemometer system used is a Dantec StreamLine $90 \mathrm{~N} 10$ frame in conjunction with a $90 \mathrm{C} 10$ constant temperature anemometer module operated at a resistance overheat of $80 \%$. An offset and gain was applied to the top of the bridge voltage in order to match the voltage range of the 16-bit A/D converter used. In order to avoid aliasing at the higher velocities, an inbuilt analog low-pass filter was set up with $10 \mathrm{kHz}$ cut-off frequency prior to the data acquisition, which was performed at $20 \mathrm{kHz}$.

\section{Disturbance generation and measurements}

The measurements of the 3D TS-instability characteristics were carried out at fully controlled disturbance conditions. The 3D velocity perturbations were excited in the airfoil boundary layer by means of a special disturbance source located at the chordwise position $x^{\prime} / c$ $=0.15\left(x^{\prime}=120 \mathrm{~mm}, x_{s}^{\prime}=131.8 \mathrm{~mm}\right)$. A prototype of this kind of source was developed and used for the first time in experiments ${ }^{23}$ devoted to investigations of the surface vibration receptivity problem at excitation of unsteady Görtler vortices. The main part of the source represented a spanwise row of circular latex membranes, mounted flush with the model surface [Fig. 1(b)] oscillating under the influence of pressure fluctuations excited by a set of speakers, playing a role of pumps. The speakers were located outside the windtunnel test section and were connected to the source by an array of plastic pipes. The spanwise spacing of the membranes was selected to be equal to $8 \mathrm{~mm}$ in the main measurements. In various regimes of measurements, either all membranes or part of them (including the important case of one membrane) were activated. In the cases of spanwise-periodic excitation, the minimum spanwise wavelength $\lambda_{z}^{\prime}$ of the surface vibrations was equal to $8 \mathrm{~mm}$. Values of $\lambda_{z}^{\prime}=32$ and $64 \mathrm{~mm}$ were used as well. Depending on the level of excitation, the membranes oscillated with amplitudes from tenth of a micrometer to several dozens of micrometers.

The excited disturbances were harmonic in time and had various frequencies and spanwise-wavenumber spectra. In these measurements, the source was used in two, basically, different ways: either with actuation of only one surface membrane (producing spanwise-localized disturbance) or with actuation of the whole row of surface membranes (producing spanwise-periodic disturbances). In the former case, the spanwise-wavenumber spectrum of the excited boundary-layer disturbances was broad, but the accuracy of measurements was relatively low due to distribution of the disturbance energy among numerous modes of the frequencywavenumber spectrum. In the latter case, the spanwise-wavenumber spectrum of the excited disturbances consisted of two or three oblique (in general) modes only, but the accuracy of measurements was much higher due to the concentration of the disturbance energy in this small number of modes. The excitation amplitude was kept at a low enough level to ensure a linear development of disturbance amplitudes.

The stability experiments with spanwise-periodic disturbances were basically performed for measurements of wall-normal profiles of the disturbance amplitudes and phases, for their comparison with eigenfunctions of the TS-instability modes, and for identification, in this way, of the physical nature of the boundary-layer perturbations. Additionally, these measurements were used for determining the wall-normal location of the maximum disturbance amplitude and for the selection of appropriate wall distances (amplitude maximum locations) used in the main instability measurements.

The stability measurements carried out for the spanwiselocalized disturbances were the main ones. Sets of spanwise distributions of disturbance amplitude were obtained at various chordwise coordinates for every excitation frequency. These data were Fouriertransformed in the spanwise direction to obtain the spanwisewavenumber spectra as well as the streamwise wavenumbers and propagation angles for every particular frequency and spanwise wavenumber. The procedure of complete decomposition of wave trains into fans of oblique waves was developed and applied for the first time for TS-waves propagating in the Blasius boundary layer in Ref. 11. Later, similar procedures were applied to other instability modes in various boundary-layer flows including the CFinstability waves developing in a swept-wing boundary layer (see, e.g., Refs. 22, 24, and 25) and unsteady Görtler vortices on concave walls. ${ }^{26}$ 
In addition, special measurements were performed to examine the linearity of the instability mechanisms under study at the particular experimental conditions (frequencies, spanwise wavenumbers, amplitudes, etc.).

The experimental data were processed, and the instability characteristics were obtained in the Fourier space for every particular harmonic of the frequency-spanwise-wavenumber spectrum.

\section{E. Theoretical approaches to base-flow and stability calculations}

\section{Base-flow calculations}

The mean flow used for stability analyses presented here was obtained through the boundary-layer computations using the pressure distribution along the model surface. The calculations were performed based on the infinite-swept wing assumption. The pressure distribution was given by CFD simulations of the flow for the experimental setup including the contoured side walls. A small mismatch between the computed and measured pressure distribution and the boundary-layer characteristics was observed. The differences can be attributed to small deviation of the angle of attack and the presence of the traverse system when stability measurements were performed. To improve the agreement between the boundary-layer characteristics from the computations and the experimental data, a small modification to the computed wall-pressure distribution was made. The comparison between the measured and the computed characteristics of the boundary layer is reported in Sec. III.

\section{Stability analysis}

The base-flow linear stability characteristics were computed using two approaches: (i) a locally parallel one and (ii) a nonlocalnonparallel approach based on the parabolized stability equations (PSE). ${ }^{18}$ The analyses are performed using the NOLOT code. ${ }^{28,29}$ The PSE computations include effects of the surface curvature, while in local stability analysis, the curvature effects have been excluded. Furthermore, to mimic the experiment as closely as possible, the amplitude of the perturbations (based on the streamwise disturbance velocity component) was evaluated at the same wall-normal position as in the experiment.

\section{MEASURED BASE-FLOW STRUCTURE}

\section{A. Potential-flow structure}

The shapes of the potential-flow streamlines measured by the wake-wire technique are presented in Fig. 2 for three spanwise positions corresponding to the upper (U), middle (M), and lower (L) wake-wires. The comparison of the shapes of these streamlines with each other is also shown along with a common parabolic approximation. It is seen that the streamline shapes are practically the same at all studied spanwise coordinates. This result supports the conclusion on the spanwise uniformity of the base flow and satisfaction of the sweep condition in the region of main stability and receptivity measurements. The streamlines are seen to be nearly straight lines in this experiment. Nevertheless, the parabolic approximation of the averaged streamline shown in Fig. 2 displays a somewhat better matching with the experimental points. The second derivative $\partial^{2} z / \partial x^{2}$ of the approximating parabola is negative, i.e.,

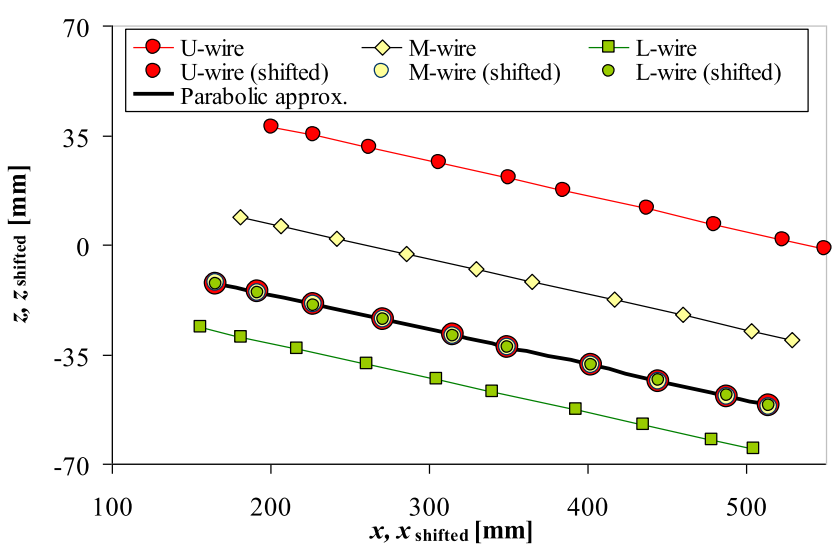

FIG. 2. Projection of streamlines of potential flow over airfoil surface measured for upper (U), middle (M), and lower (L) wake-wires and a comparison of their shapes after shifting the streamwise and spanwise coordinates.

the streamline is slightly convex, and the flow-velocity vectors are turning slightly in the direction opposite to that of the $z$-axis.

Main results of measurements of the $U_{e}^{\prime}$ - and $W_{e}^{\prime}$-components of the inviscid mean-velocity vector are presented in Fig. 3(a). The chordwise distributions of the two velocity components, taken just outside the boundary-layer edge (at $y \approx 10 \mathrm{~mm}$ ) at three spanwise locations, display a good agreement with each other. This fact, together with the constancy of the spanwise $W_{e}^{\prime}$-component, proves that the base-flow under study complies well with the assumption of an infinite-span swept wing.

In a set of special single-wire experiments, three streamwise distributions of the streamwise velocity component $\left(U_{e}\right)$ of the potential-flow velocity vector were directly measured outside the boundary-layer edge (at $y \approx 10 \mathrm{~mm}$ ) at three spanwise locations: $z_{c}^{\prime}=+60,0$, and $-60 \mathrm{~mm}$. The results are presented in Fig. $3(\mathrm{~b})$ in comparison with the corresponding calculated distribution. A very good agreement between the measured and the calculated distributions is observed. It is seen that, in contrast to the first part of the present study, ${ }^{1}$ the streamwise [Fig. 3(b)] and the chordwise [Fig. 3(a)] velocity components depend very weakly on the chordwise coordinate. This condition guarantees development and predominance of the TS-instability mechanism since the CF-instability can hardly appear due to the absence of any significant chordwise favorable pressure gradient. This conclusion is supported by our stability analysis.

\section{B. Boundary-layer flow}

The measured and calculated wall-normal profiles of the streamwise component of the mean-velocity vector are illustrated in Figs. 4(a) and 4(b) for two chordwise positions and three spanwise positions. Obviously, the experimental and theoretical profiles are in good agreement. The experimental velocity profiles do not display any spanwise dependency, supporting the spanwise homogeneity of the boundary layer in the region of main measurements. The corresponding crossflow velocity profiles calculated for the same two chordwise positions are presented in Fig. 4(c). It is seen 

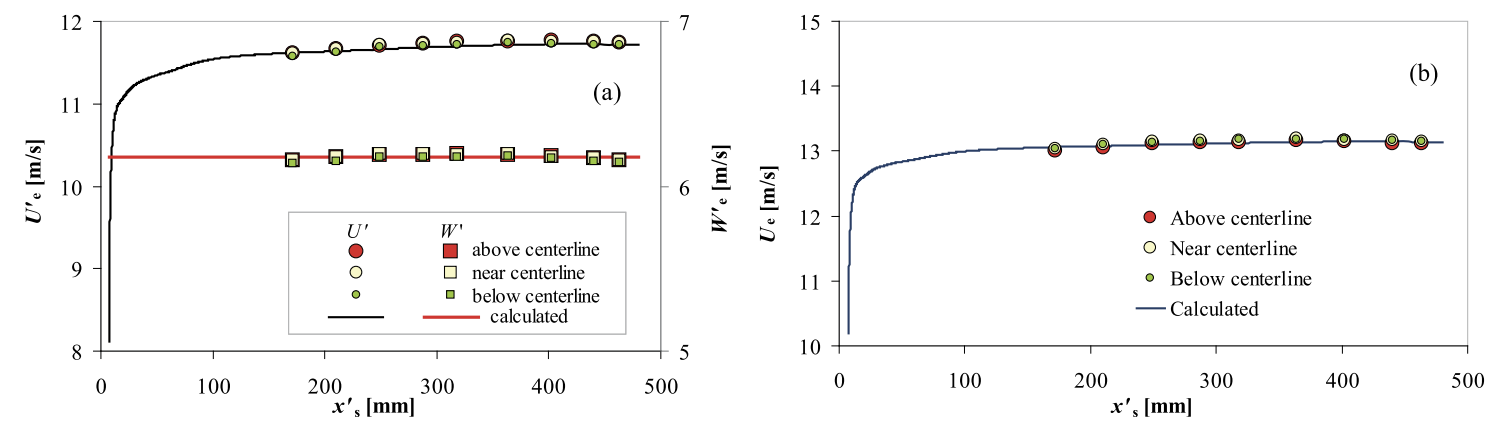

FIG. 3. Chordwise distributions of chordwise $\left(U_{e}^{\prime}\right)$ and spanwise $\left(W_{e}^{\prime}\right)$ components of the potential-flow velocity vector measured for three spanwise locations near the boundary-layer edge (a) and a comparison of the measured and calculated chordwise distributions of the streamwise component $\left(U_{e}\right)$ of the potential-flow velocity vector (b). The measurements were performed at a practically constant wall-normal coordinate of $y \approx 10 \mathrm{~mm}$.

that the crossflow is very weak in this case even at the disturbance source chordwise location and decays downstream. Such crossflow intensity can hardly lead to appearance of the CF-instability. However, its presence is important and leads to a significant asymmetry
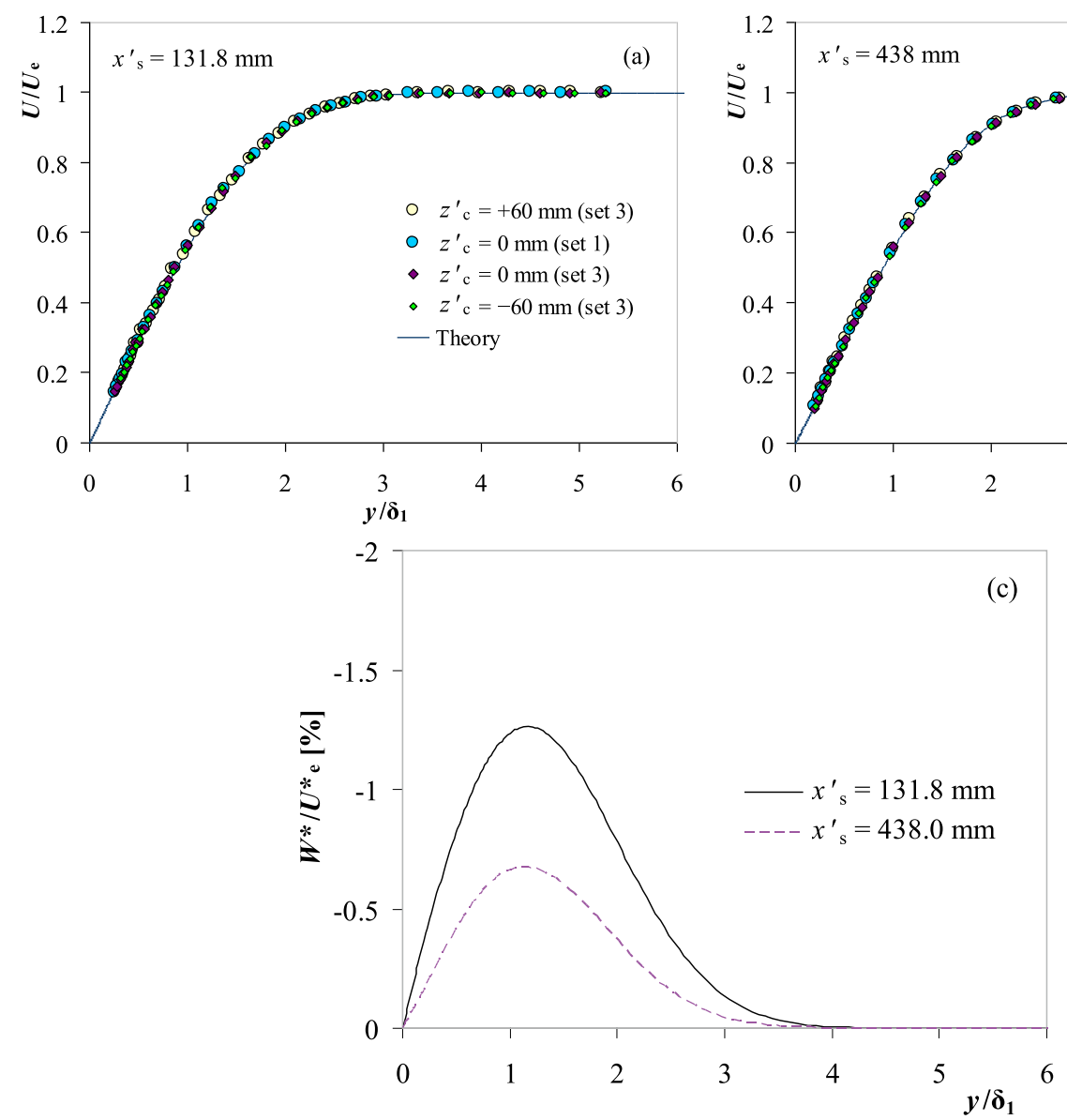

of the TS-wave growth-rate distributions vs the wave propagation angle discussed below, as well as to asymmetry of the corresponding dispersion characteristics in comparison with the $2 \mathrm{D}$ boundary layers.

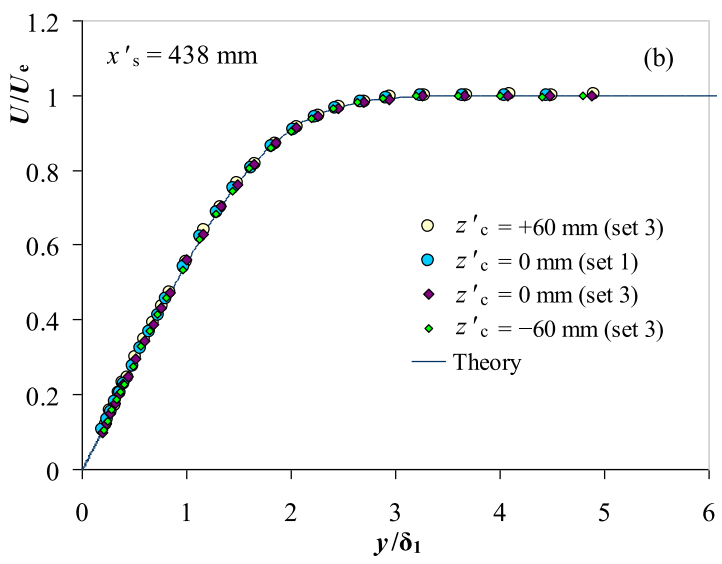

(c)

6

FIG. 4. Wall-normal profiles of the streamwise mean-velocity component measured at two chordwise and three spanwise locations in comparison with the calculated ones. (a) $x_{s}^{\prime}=131.8 \mathrm{~mm}$ (source position) and (b) $x_{s}^{\prime}=438 \mathrm{~mm}$ (last measured chordwise position). Cross-flow mean-velocity profiles calculated for the same two chordwise positions are shown in plot (c). 


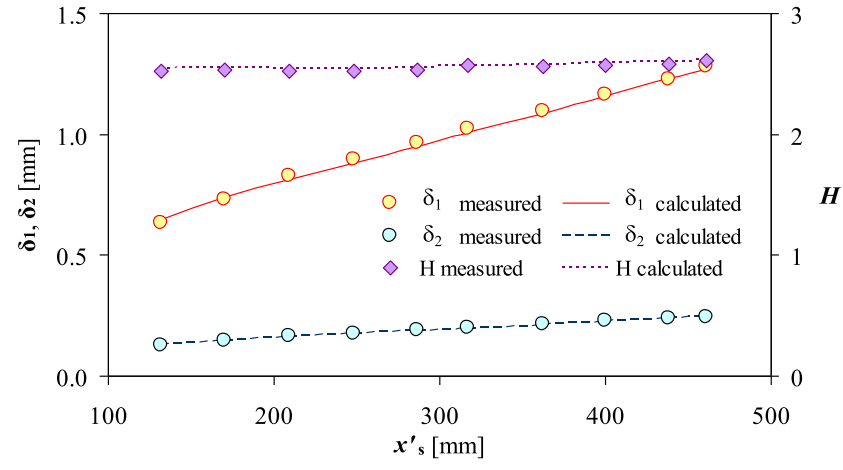

FIG. 5. Boundary-layer displacement thicknesses and shape factor measured for the streamwise mean-velocity component vs chordwise coordinate $x_{s}^{\prime}$ in comparison with calculated ones.

The experimental values of the boundary-layer displacement thickness $\delta_{1}$, momentum thickness $\delta_{2}$, and shape factor $H=\delta_{1} / \delta_{2}$ were found by means of integration of the profiles like those shown in Figs. 4(a) and 4(b). They are presented in Fig. 5 in comparison with the calculated ones. A good agreement between the measured and calculated data is observed for all three boundary-layer parameters. It is seen, in particular, that the shape factor increases only slightly in the downstream direction and in the last three experimental points reach values, which are very close to the value typi$\mathrm{cal}$ for the Blasius boundary layer $(H=2.591)$. Note, however, that the boundary layer remains three-dimensional in the whole region of measurements. This circumstance influences the TS-instability characteristics obtained and discussed below.

\section{BOUNDARY-LAYER DISTURBANCES AND THEIR STREAMWISE EVOLUTION}

\section{A. Regimes of TS-instability measurements}

During the stability measurements, the $3 \mathrm{D}$ TS-waves were generated by the surface disturbance source described above. In the case of spanwise-localized excitation of the TS wave trains, the spanwise wavenumber spectrum of the generated modes was broadband, while in the case of spanwise-periodic excitation, only two or three modes (with spanwise wavenumbers $\pm \beta_{1}^{\prime}$ and, sometimes, $\beta^{\prime}=0$ ) were excited.

Ranges of the studied disturbance frequencies and spanwise wavenumbers were selected based on the linear-stability calculations performed at a stage of preparation of the experiments. Later, these calculations have been improved based on the measured base flow characteristics. Contours of the calculated TS-wave amplification factors ( $N$ factors) for chordwise locations $x_{s}^{\prime}=209$ and $438 \mathrm{~mm}$ are shown in Fig. 6. The TS-mode frequencies selected for performing main stability measurements are marked there with vertical lines. These frequencies were 80, 120, 156, 194, and $234 \mathrm{~Hz}$. The majority of these frequencies correspond to TS-modes which are either amplified ones or neutrally stable ones or close to neutrally stable ones in the studied chordwise range. Close to the disturbance source, the amplified modes have rather high frequencies and only positive spanwise wavenumbers. Further downstream, the modes with relatively lower frequencies having zero and negative spanwise wavenumbers can be amplified as well. Note that the diagram shown in Fig. 6 differs very significantly from that calculated for the CF-instability modes, as shown in Fig. 5 in Ref. 1. In particular, the most amplified TS-waves have significantly higher frequencies compared to the CF-modes and also the TS-wave instability region excludes the zero-frequency perturbations. In addition, the CF-modes with the zero spanwise wavenumber always attenuate, while the TS-modes with $\beta^{\prime}=0$ can be amplified.

The surface disturbance source had a spanwise spacing of the membranes of $\lambda_{z}^{\prime}=8 \mathrm{~mm}$ corresponding to the maximum possible spanwise wavenumber of the spanwise-periodically excited disturbances $\beta_{\text {max }}^{\prime}= \pm 2 \pi / \lambda_{z}^{\prime}= \pm 0.785 \mathrm{rad} / \mathrm{mm}$. However, we excited basically the TS-modes with significantly lower spanwise wavenumbers, which corresponded to the amplified disturbances. In the experiments with excitation of spanwise-periodic perturbations, the following spanwise wavenumbers were selected $\pm \beta_{1}^{\prime}= \pm 0.098 \mathrm{rad} / \mathrm{mm}$ or $\pm \beta_{2}^{\prime}= \pm 0.196 \mathrm{rad} / \mathrm{mm}$ or $\pm \beta_{0}^{\prime}=0$. These modes are also
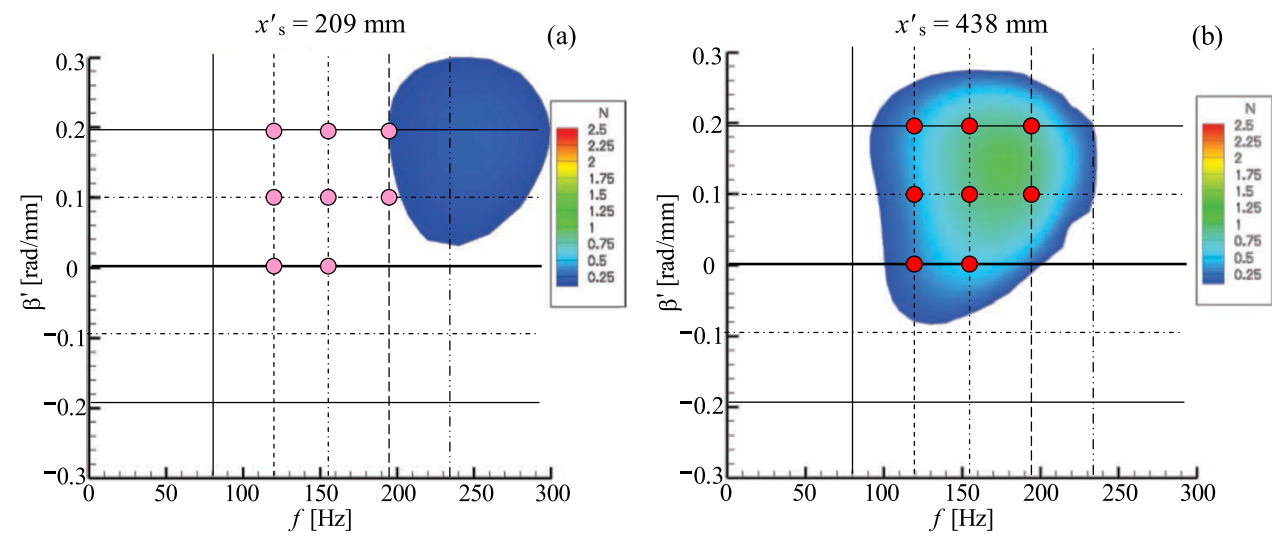

FIG. 6. Linear-stability diagrams (contours of $\mathrm{N}$-factors) calculated for streamwise positions $x_{\mathrm{s}}^{\prime}=209$ (a) and 438 (b) mm. Studied disturbance frequencies are marked with vertical lines. Values of spanwise wavenumbers selected for main measurements of wall-normal profiles (eigenfunctions) are marked with horizontal lines. Circles display points of frequency-wavenumber spectrum for which wall-normal profiles have been measured. 
TABLE I. Studied TS-instability regimes with excitation of spanwise-periodic disturbances.

\begin{tabular}{|c|c|c|c|c|c|c|}
\hline Regime & $f_{\text {sur }}(\mathrm{Hz})$ & $\lambda_{z}^{\prime}(\mathrm{mm})$ & Phase of excitation & $\beta^{\prime}$ excited $(\mathrm{rad} / \mathrm{mm})$ & $E$ & $\beta^{\prime}$ in the flow $(\mathrm{rad} / \mathrm{mm})$ \\
\hline ZPF2B0(A9) & 120 & 8 and $\infty$ & All in phase & \pm 0.785 and 0 & 9.0 & 0 \\
\hline ZPF2B0(A5) & 120 & 8 and $\infty$ & All in phase & \pm 0.785 and 0 & 5.0 & 0 \\
\hline ZPF2B1(A8) & 120 & 64 & Quartets in antiphase & \pm 0.098 & 8.0 & \pm 0.098 \\
\hline ZPF2B1(A6) & 120 & 64 & Quartets in antiphase & \pm 0.098 & 6.0 & \pm 0.098 \\
\hline ZPF2B1(A4) & 120 & 64 & Quartets in antiphase & \pm 0.098 & 4.0 & \pm 0.098 \\
\hline ZPF2B1(A3) & 120 & 64 & Quartets in antiphase & \pm 0.098 & 3.0 & \pm 0.098 \\
\hline ZPF2B3(A9) & 118 & 32 & Pairs in antiphase & \pm 0.196 & 9.0 & +0.196 \\
\hline ZPF3B0(A5) & 156 & 8 and $\infty$ & All in phase & \pm 0.785 and 0 & 5.0 & 0 \\
\hline ZPF3B0(A3) & 156 & 8 and $\infty$ & All in phase & \pm 0.785 and 0 & 3.0 & 0 \\
\hline ZPF3B1(A4) & 156 & 64 & Quartets in antiphase & \pm 0.098 & 4.0 & \pm 0.098 \\
\hline ZPF3B3(A9) & 156 & 32 & Pairs in antiphase & \pm 0.196 & 9.0 & +0.196 \\
\hline ZPF4B1(A1.5) & 194 & 64 & Quartets in antiphase & \pm 0.098 & 1.5 & \pm 0.098 \\
\hline ZPF4B1(A3) & 194 & 64 & Quartets in antiphase & \pm 0.098 & 3.0 & \pm 0.098 \\
\hline ZPF4B3(A2) & 194 & 32 & Pairs in antiphase & \pm 0.196 & 2.0 & +0.196 \\
\hline
\end{tabular}

indicated in Fig. 6 by horizontal lines. The relative shift $\Delta \varphi_{m}$ between initial phases of oscillations of all membranes was equal to zero in the case of $\beta_{0}^{\prime}$ (i.e., all membranes oscillated in-phase). Meanwhile, at excitation of modes $\pm \beta_{2}^{\prime}$, this shift was equal to zero for one pair of neighboring membranes, but it was equal to $180^{\circ}$ between every two neighboring pairs of membranes. In the case of excitation of modes with spanwise wavenumbers of $\pm \beta_{1}^{\prime}, \Delta \varphi_{m}$ was equal to zero for four neighboring membranes, but it was equal to $180^{\circ}$ between every two neighboring quartets of membranes. Of course, in the case of localized excitation with only one membrane actuated, the spanwise-wavenumber range of the excited TS-modes was much broader and included all values of the spanwise wavenumber shown in Fig. 6.

The 3D TS-instability problem has been studied for the following controlled parameters: (i) frequency of surface nonuniformities and excited TS-waves, (ii) spanwise wavenumber (or scale) of surface nonuniformities and excited TS-waves, and (iii) amplitude E of membrane excitation controlled by a computer program used for signal generation measured in relative units. The complete list of the studied regimes is presented in Tables I and II.

TABLE II. Studied TS-instability regimes with excitation of spanwise-localized disturbances.

\begin{tabular}{lccc}
\hline \hline Regime & $f_{\text {sur }}(\mathrm{Hz})$ & $E$ & $U / U_{e}$ \\
\hline ZLF1(A3) & 80 & 3.0 & 0.435 \\
ZLF1(A8) & 80 & 8.0 & 0.435 \\
ZLF1(A9) & 80 & 9.0 & 0.435 \\
ZLF2(A6) & 120 & 6.0 & 0.435 \\
ZLF3(A3) & 156 & 3.0 & 0.435 \\
ZLF3(A6) & 156 & 6.0 & 0.435 \\
ZLF4(A6) & 194 & 6.0 & 0.435 \\
ZLF5(A6) & 234 & 6.0 & 0.435 \\
\hline \hline
\end{tabular}

\section{B. Characteristics of boundary-layer disturbances}

In order to investigate the physical nature of the excited boundary-layer disturbances, a set of special tests has been performed in regimes with excitation of spanwise-periodic perturbations (see Table I). These measurements were carried out with excitation of TS-waves at three pairs of values of the spanwise wavenumber: either $0, \pm 0.098$, or $\pm 0.196 \mathrm{rad} / \mathrm{mm}$ for three frequencies $f=120,156$, or $194 \mathrm{~Hz}$. The most interesting frequencywavenumber pairs, which have survived at the end of the region of measurements, are indicated in Fig. 6 with magenta and red circles. These measurements were used, in particular, to obtain the wallnormal profiles of disturbance amplitudes and phases. These data were also used to find the nondimensional wall-normal location of the TS-wave amplitude maximum to be used in the main stability measurements.

Shown in Fig. 7 are three examples of the spanwise distributions of amplitudes and phases of boundary-layer disturbances for frequency $f=156 \mathrm{~Hz}$ measured at $x_{s}^{\prime}=438 \mathrm{~mm}$ and $y / \delta_{1}$ $=0.77$. The data correspond to regimes ZPF3B0(A3), ZPF3B3(A9), and ZPF3B1(A4) for spanwise wavenumbers $\beta^{\prime}=0,+0.196$, and $\pm 0.098 \mathrm{rad} / \mathrm{mm}$, respectively. Here, $A$ is the $\mathrm{rms}$ amplitude of the streamwise velocity disturbances normalized by local boundarylayer edge velocity $U_{e}$. The wall-normal profiles were measured at spanwise locations marked in these figures with vertical red lines. Four of them, corresponding to Figs. 7(a) and 7(b), are presented in Figs. 8(a) and 8(b) and discussed below.

It is seen that the amplitude and phase distributions shown in Fig. 7(a) do correspond to predominance of a single mode with $\beta^{\prime}=0$. This mode has constant amplitude and phase in the spanwise direction. Two other modes excited by the source in regime ZPF3B0(A3) at $\beta^{\prime}= \pm 0.785 \mathrm{rad} / \mathrm{mm}$ (see Table I) have decayed at this late chordwise position because they are far away from the instability region (see Fig. 6). A similar situation is observed in regime ZPF3B3(A9) [Fig. 7(b)], in which one of two excited modes having spanwise wavenumber $\beta^{\prime}=-0.196 \mathrm{rad} / \mathrm{mm}$ has decayed at the chordwise location $x_{s}^{\prime}=438 \mathrm{~mm}$ (due to action of the 

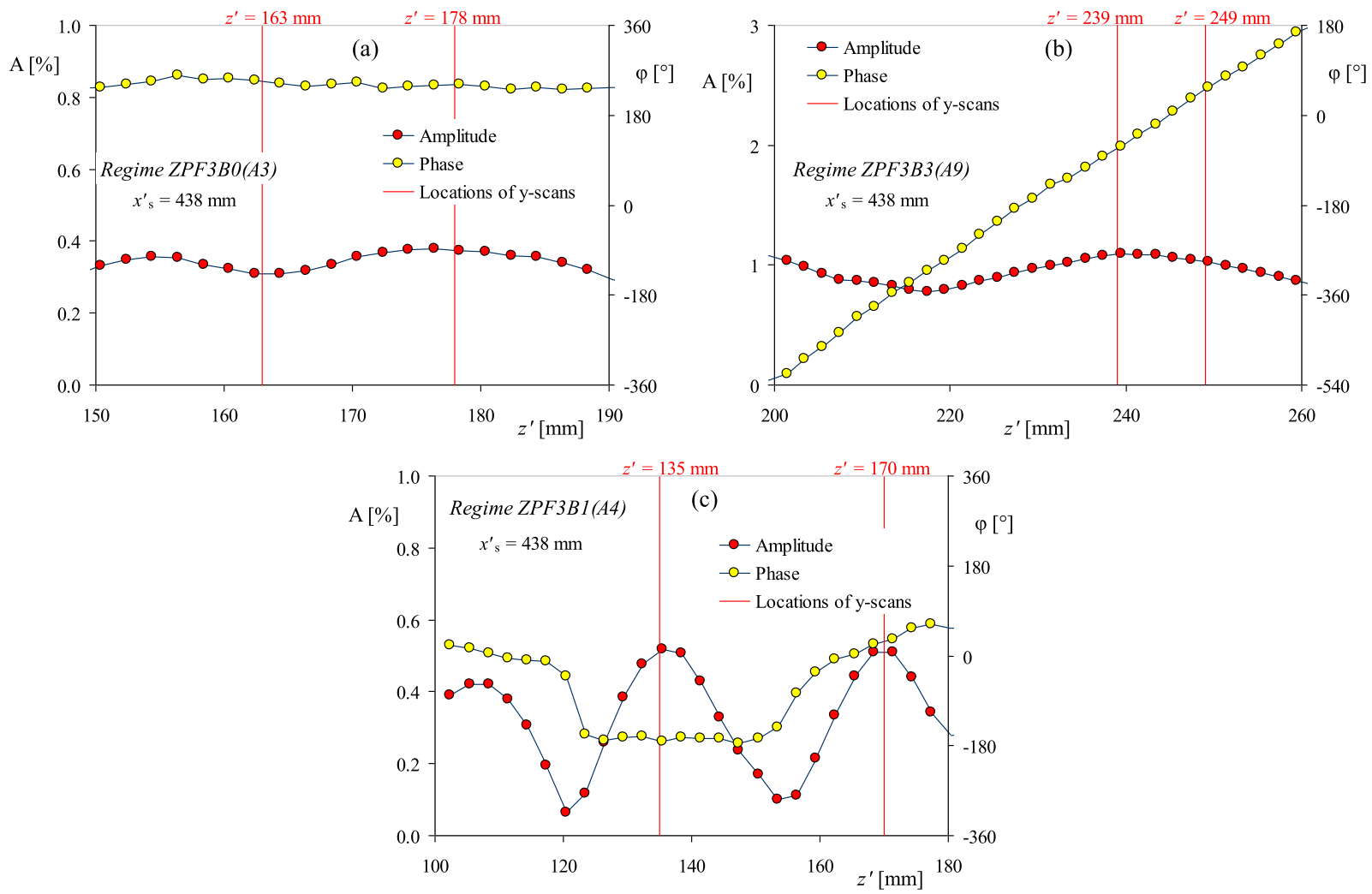

FIG. 7. Spanwise distributions of amplitudes and phases of boundary-layer disturbances measured for frequency $f=156 \mathrm{~Hz}$ in regimes ZPF3B0(A3) (a), ZPF3B3(A9) (b) and ZPF3B1(A4) (c) for spanwise wavenumbers $\beta^{\prime}=0,+0.196$, and $\pm 0.098 \mathrm{rad} / \mathrm{mm}$, respectively. The data were measured at $y / \delta_{1}=0.77$ and $x_{s}^{\prime}=438 \mathrm{~mm}$.

linear stability mechanism, as seen in Fig. 6) and the measured spanwise distributions of the disturbance amplitudes and phases [Fig. 7(b)] correspond, basically, to the only remaining mode with $\beta^{\prime}=+0.196 \mathrm{rad} / \mathrm{mm}$ with nearly constant amplitude and linearly growing phase. Meanwhile, the situation is significantly different in regime $\mathrm{ZPF} 3 \mathrm{~B} 1(\mathrm{~A} 4)$ [Fig. $7(\mathrm{c})$ ]. In this case, none of the two excited modes (with $\beta^{\prime}= \pm 0.098 \mathrm{rad} / \mathrm{mm}$ ) decay significantly downstream (see Fig. 6). Consequently, they both remain present in the
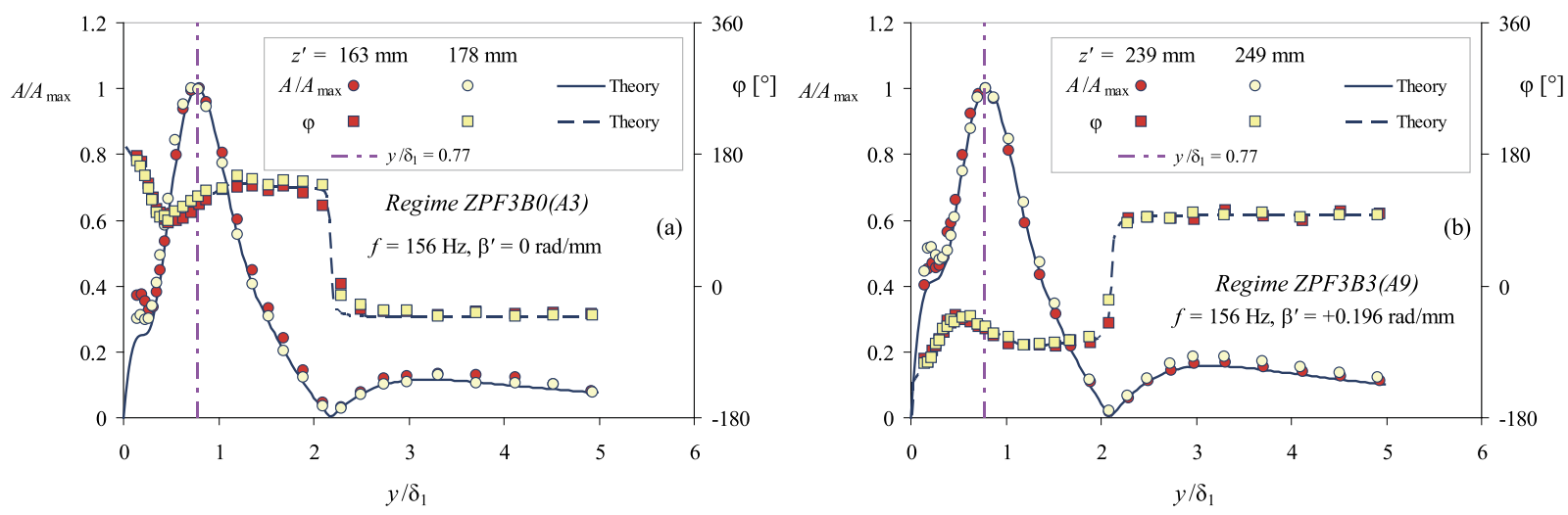

FIG. 8. Wall-normal profiles of amplitudes and phases of boundary-layer disturbances measured in regimes ZPF3BO(A3) (a) and ZPF3B3(A9) (b) for spanwise wavenumbers $\beta^{\prime}=0$ and $+0.196 \mathrm{rad} / \mathrm{mm}$, respectively, at $x_{s}^{\prime}=438 \mathrm{~mm}$ at two spanwise locations (symbols) in comparison with calculated amplitudes and phases of eigenfunctions of normal TS-instability modes (lines). 
boundary layer and display a typical standing-wave picture with modulated amplitude and jumping phase.

Examples of wall-normal profiles of amplitudes and phases of boundary-layer disturbances are presented in Figs. 8(a) and 8(b), respectively. These data were measured in regimes $\mathrm{ZPF} 3 \mathrm{~B} 0(\mathrm{~A} 3)$ and ZPF3B3(A9) at $x_{s}^{\prime}=438 \mathrm{~mm}$ and two different spanwise locations [marked in Figs. 7(a) and 7(b) with vertical red lines]. A comparison of these profiles with calculated amplitudes and phases of eigenfunctions of the corresponding normal TS-instability modes shows a good agreement. This confirms that the boundary-layer disturbances excited and measured in the present experiment correspond to $3 \mathrm{D}$ TS-waves. The analysis of all studied wall normal profiles showed that the distance corresponding to the dimensionless streamwise mean velocity $U / U_{e}=0.435$ is the best compromise for the location of the maximum disturbance amplitude for all TS-instability waves in the studied range of parameters. This distance is indicated in Figs. 8(a) and 8(b) by the vertical red lines. The value $U / U_{e}=0.435$ corresponds to $y / \delta_{1}=0.77$ and turned out to be the same as that selected in several previous investigations performed in $2 \mathrm{D}$ boundary layers for various 3D TS-waves (see, e.g., Refs. 13 and 30 for experiments with the Blasius flow). Therefore, this value of the dimensionless wallnormal distance was used at measurements of spanwise distributions in all stability and receptivity experiments with excitation of TS-waves.

\section{Linearity of TS-instability problem under study}

The independence of the measured TS-instability characteristics from the disturbance amplitude was investigated in two pairs of regimes: ZPF2B1(A6) and ZPF2B1(A3) (Table I) and ZLF3(A6) and ZLF3(A3) (Table II). All parameters of the base flow and the excited disturbances were the same within every pair of regimes except for the amplitudes of the excited modes. The level of excitation (a relative amplitude $E$ ) was reduced in regimes ZPF2B1(A3) and ZLF3(A3) by a factor of two compared to regimes ZPF2B1(A6) and ZLF3(A6).
Shown in Figs. 9(a) and 9(b) are the wall-normal distributions of the boundary-layer disturbance amplitudes and phases measured in regimes ZPF2B1(A6) and ZPF2B1(A3) for $f=120 \mathrm{~Hz}$ at $x^{\prime} / c=0.531\left(x_{s}^{\prime}=438 \mathrm{~mm}\right)$, i.e., far downstream of the disturbance source. At these conditions, two modes with spanwisewavenumber $\beta^{\prime}= \pm 0.098 \mathrm{rad} / \mathrm{mm}$ are present in the flow. The same amplitude profiles but normalized by their maximum are also shown in Fig. 9(a). It is clear that the shapes of both the amplitude and phase profiles are the same for the two studied levels of excitation, i.e., they both are independent of the disturbance amplitude. They exhibit the typical shape of oblique TS-wave eigenfunctions, which have been investigated in detail in experiments ${ }^{13}$ in the Blasius boundary layer and are shown, in particular, in Figs. 12 and 13 in that paper.

Two pairs of spanwise distributions of disturbance amplitudes and phases shown in Figs. 10(a) and 10(b) were measured inside the boundary layer (at $U / U_{e}=0.435$ ) at two chordwise coordinates: $x^{\prime} / c$ $=0.198\left(x_{s}^{\prime}=171 \mathrm{~mm}\right)$ and $x^{\prime} / c=0.484\left(x_{s}^{\prime}=400 \mathrm{~mm}\right)$, i.e., at the initial chordwise position and close to the end of the region of main stability measurements, respectively. (The phases are plotted with an arbitrary constant shift $\Delta \phi=2 k \pi$ between the pairs of distributions obtained at different chordwise locations.) The distributions measured at $x^{\prime} / c=0.484$ normalized by the spanwise-averaged values of disturbance amplitudes and phases obtained at the initial section $x^{\prime} / c=0.198$ are shown in Figs. 10 (a) and 10 (b) as well. The averaging was performed only for those points in which the accuracy of measurements was reliable due to large enough disturbance amplitudes. The amplitude distributions directly measured at $x^{\prime} / c=0.484$ were divided by the corresponding averaged amplitude measured at $x^{\prime} / c=0.198$, while the average phases measured at $x^{\prime} / c=0.198$ were subtracted from the phase distributions directly measured at $x^{\prime} / c$ $=0.484$. As a result of such normalization, the normalized amplitude and phase distributions shown in Figs. 10(a) and 10(b) reflect purely the characteristics of development of the boundary-layer disturbances.

The coincidence of these normalized distributions obtained in regime $\mathrm{ZLF3}(\mathrm{A} 3)$ with those measured in regime ZLF3(A6)
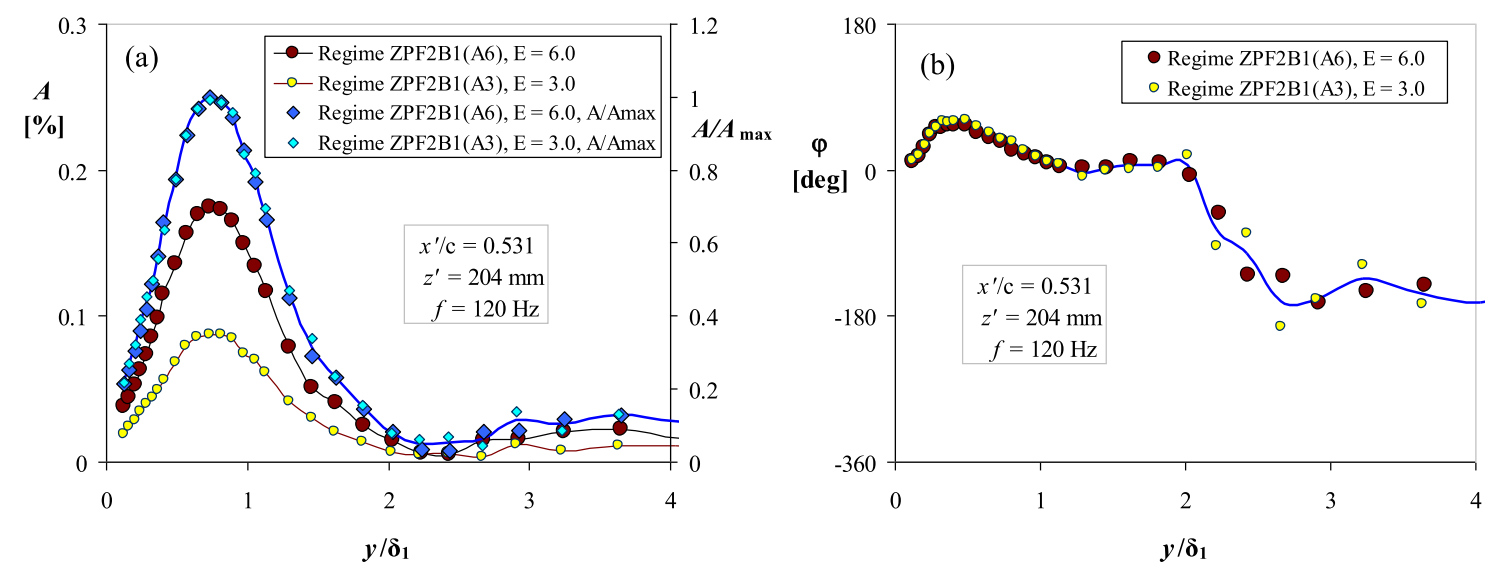

FIG. 9. Wall-normal distributions of disturbance amplitudes and normalized amplitudes (a) and phases (b) measured in regimes ZPF2B1(A6) and ZPF2B1(A3) for two amplitudes of excitation of a row of membranes. $f=120 \mathrm{~Hz}, \mathrm{E}=6$ and 3 , and $x^{\prime} / c=0.531$. 

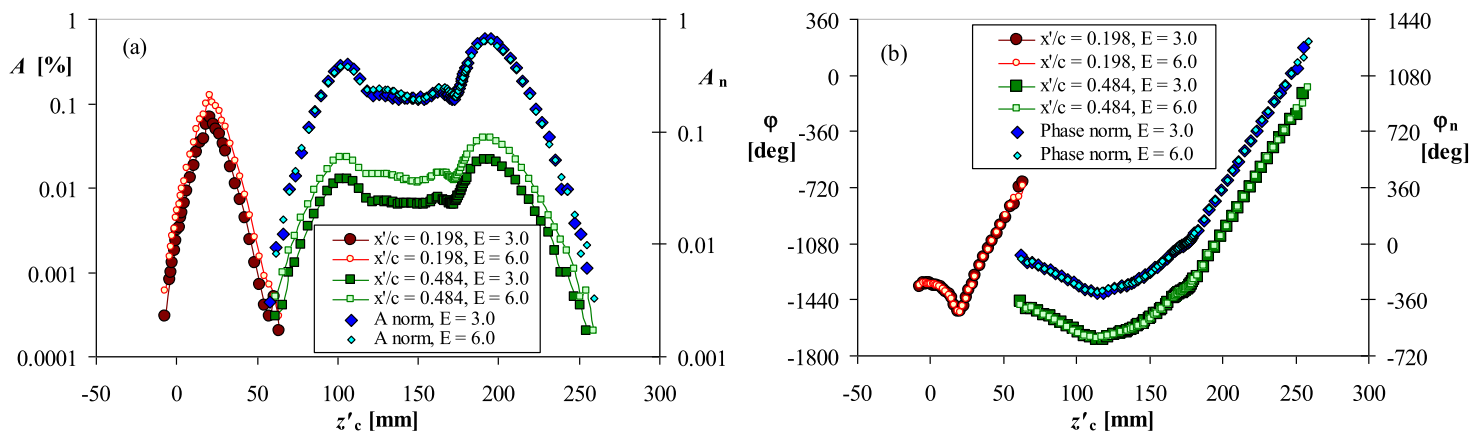

FIG. 10. Spanwise distributions of directly measured and normalized disturbance amplitudes (a) and phases (b) taken in regimes ZLF3(A6) and ZLF3(A3) for two amplitudes of excitation of single membrane (the center one). $f=156 \mathrm{~Hz}, E=6$ and 3 , and $x^{\prime} / c=0.198$ and 0.484 .

indicates the independence of the instability characteristics from the amplitude of the excited perturbations, i.e., to the linearity of the instability problem under study.

\section{Evolution of TS-wave trains}

For each selected frequency, linear stability measurements were performed for a broad range of spanwise wavenumbers. These measurements were carried out with only one oscillating membrane of the surface disturbance source, the center one (see Table II). The obtained results are illustrated below for three frequencies $f=194$, 156 , and $120 \mathrm{~Hz}$, which are close to the most amplified 3D TS-waves [see Fig. 6(b)].

An example of a set of spanwise distributions of the disturbance amplitudes and phases within a wave-train of TS-instability modes measured in regime ZLF4(A6) at frequency $f=194 \mathrm{~Hz}$ is presented in Fig. 11. It is seen that the shapes of the distributions are significantly different from those obtained in CF-instability experiments. ${ }^{1}$ In particular, the TS-wave train spreads in the spanwise direction much faster than any train consisted of CF-modes.
Similar sets of spanwise distributions were obtained in all other studied regimes with localized excitation of TS-instability modes indicated in Table II.

\section{E. Spanwise-wavenumber spectra and evolution of spectral TS-modes}

Spanwise Fourier decomposition of the distributions like those shown in Fig. 11 gave us the TS-wave spanwise-wavenumber spectra. An illustration of their amplitude parts is presented in Fig. 12 for a frequency of $f=194 \mathrm{~Hz}$ [regime ZLF4(A6)]. The corresponding phases have been found as well. In total, such spectra were obtained for every particular value of the spanwise wavenumber $\beta^{\prime}$ for all five studied frequencies (see Table II).

The downstream evolution of every particular TS-mode can be obtained experimentally as a cross section of a data set like that shown in Fig. 12 by plane $\beta^{\prime}=$ const. Examples of such cross sections are presented in Figs. 13(b) and 13(c), Figs. 14(b) and 14(c), and Figs. 15(b) and 15(c) for $f=194,156$, and $120 \mathrm{~Hz}$, respectively, for five selected values of the spanwise wavenumber $\beta^{\prime}=-0.367,0$,
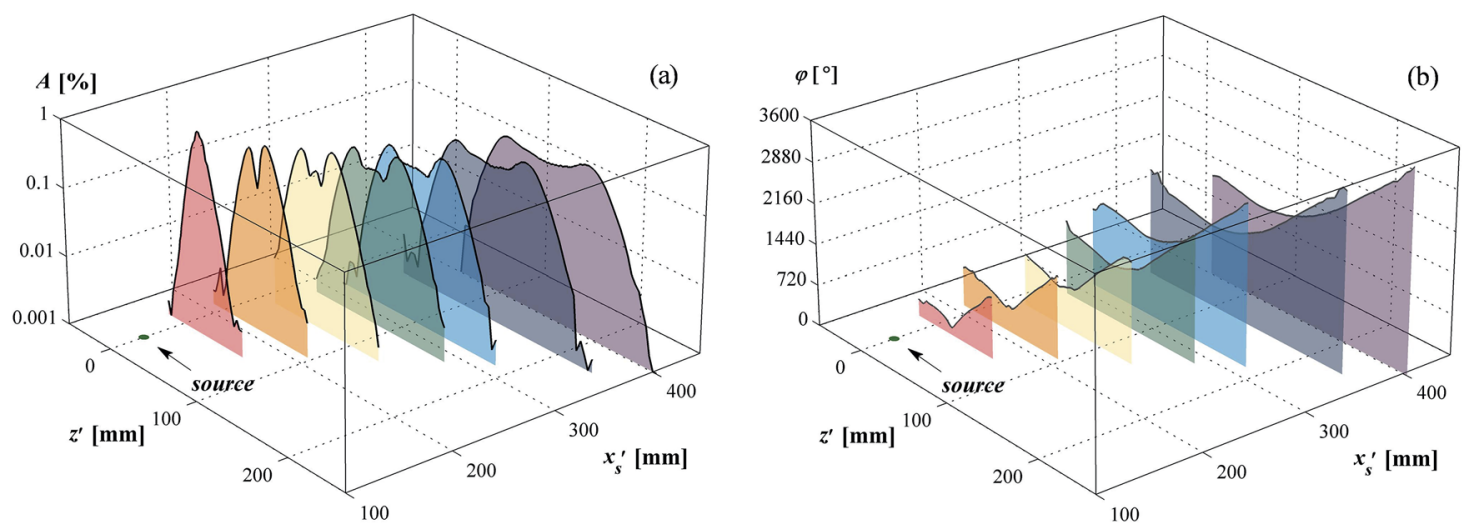

100

FIG. 11. Set of spanwise distributions of amplitudes (a) and phases (b) of disturbances within a wave-train of TS-instability modes measured in regime ZLF4(A6) at frequency $f=194 \mathrm{~Hz}$ for spanwise-localized excitation. 


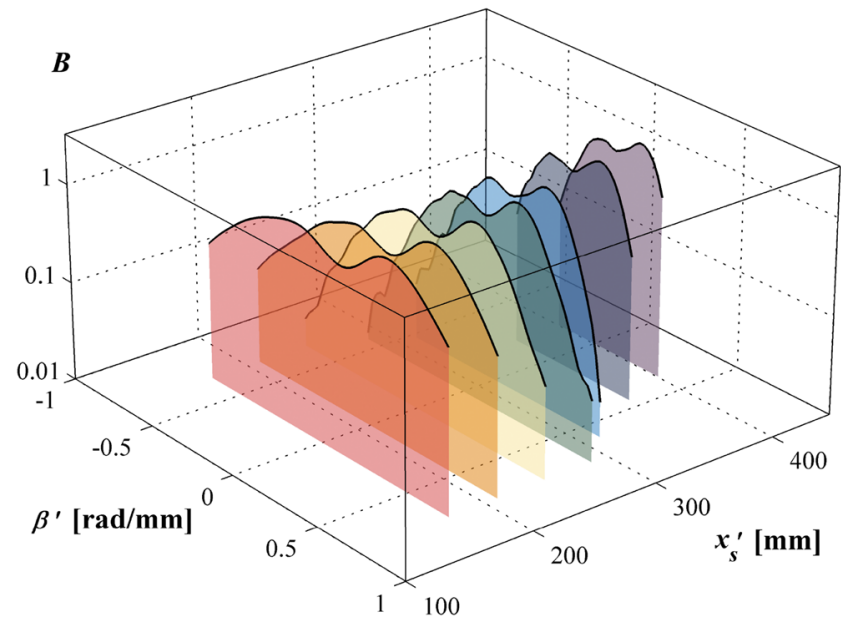

FIG. 12. Set of amplitude parts of spanwise-wavenumber spectra of the TS-waves constituting the wave-train shown in Fig. 11 measured in regime ZLF4(A6) at frequency $f=194 \mathrm{~Hz}$ for spanwise-localized excitation.

$+0.157,+0.367$, and $0.524 \mathrm{rad} / \mathrm{mm}$ marked in Figs. 13(a), 14(a), and 15(a) by vertical lines. The corresponding theoretical distributions are shown in Figs. 13(b), 13(c), 14(b), 14(c), 15(b), and 15(c) with lines.
Note first of all that the computed (based on PSE) evolution of disturbance amplitudes [Figs. 13(b), 14(b), and 15(b)] and phases [Figs. 13(c), 14(c), and 15(c)] agree well with the corresponding experimental data.

The amplification curves presented in Fig. 13(b) [regime ZLF4(A6), $f=194 \mathrm{~Hz}]$ show that modes with $\beta^{\prime}=-0.367$ and 0.524 $\mathrm{rad} / \mathrm{mm}$ attenuate quickly downstream with similar rates although their phases have completely different behavior [Fig. 13(c)]. The mode with $\beta^{\prime}=+0.367 \mathrm{rad} / \mathrm{mm}$ has initially nearly neutral behavior but attenuates slightly further downstream [Fig. 13(b)], while the modes with $\beta^{\prime}=0$ and $+0.157 \mathrm{rad} / \mathrm{mm}$ [Fig. 13(b)] are amplified in the whole range of studied chordwise coordinates. Note also that although the shape of the surface nonuniformity (the membrane) is axisymmetric, the spanwise-wavenumber spectra of the excited TSwaves are not symmetric at all. This is natural for the 3D swept-wing boundary layer in contrast to any $2 \mathrm{D}$ one.

A qualitatively similar behavior of the normal-mode amplitudes and phases with the same spanwise wavenumbers is observed in regime ZLF3(A6) with $f=156 \mathrm{~Hz}$ [Fig. 14(b)] although the disturbance amplitude attenuation and amplification rates are weaker now and the modes with $\beta^{\prime}=0$ and $+0.157 \mathrm{rad} / \mathrm{mm}$ [Fig. 14(b)] decay initially and only later start to grow.

In regime ZLF2(A6) with $f=120 \mathrm{~Hz}$, all spectral modes attenuate initially [Fig. 15(b)]. However, for $\beta^{\prime}=0$ and $+0.157 \mathrm{rad} / \mathrm{mm}$, this attenuation changes downstream of $x_{s}^{\prime} \approx 300 \mathrm{~mm}$ to neutral behavior and, then, to a weak amplification.
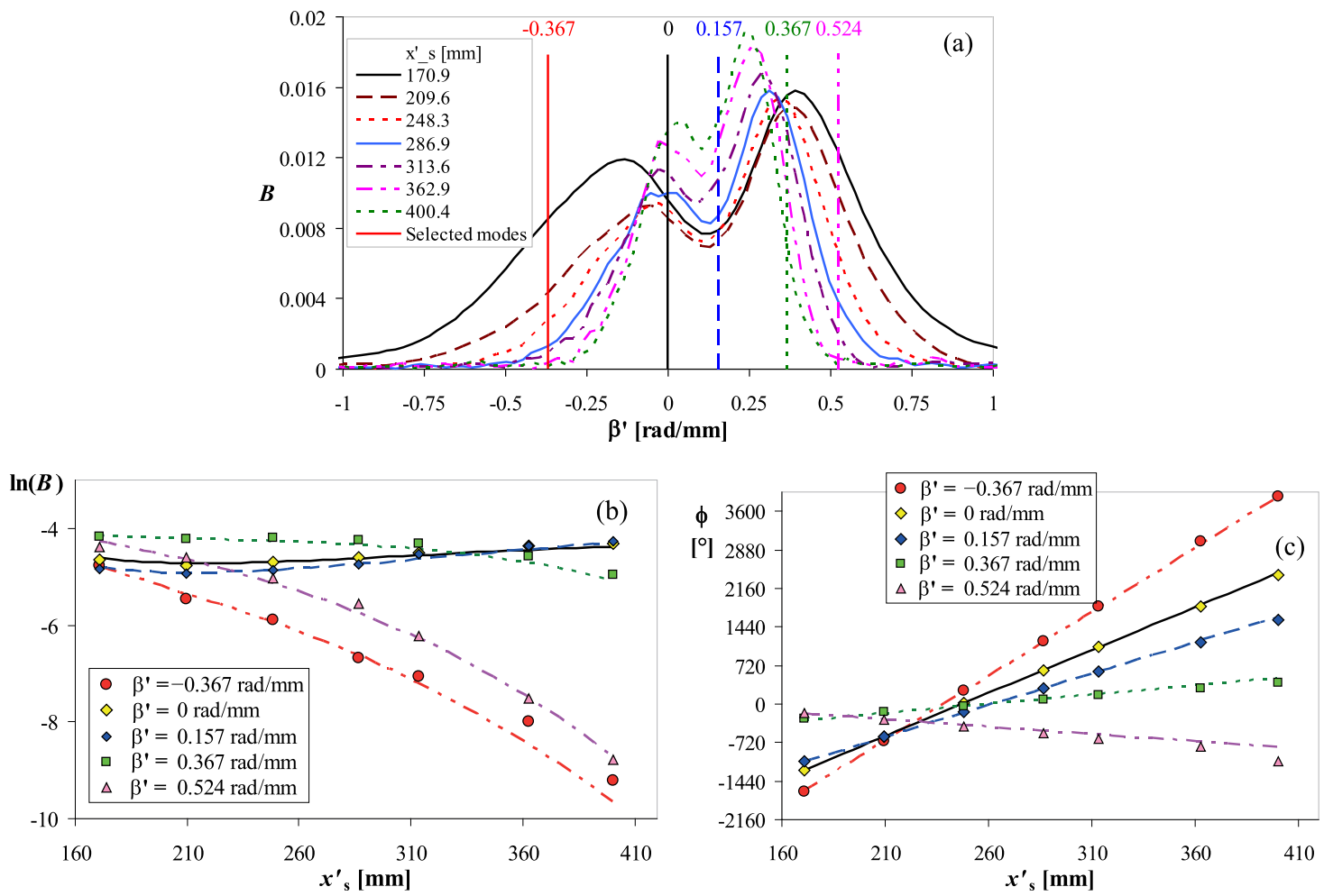

FIG. 13. Set of chordwise distributions of disturbance spectral amplitudes (b) and spectral phases (c) obtained experimentally (symbols) for $f=194 \mathrm{~Hz}$ in regime ZLF4(A6) from spectra shown in plot (a) at selected spanwise wavenumbers indicated by vertical lines. Corresponding theoretical distributions are shown with various lines. 

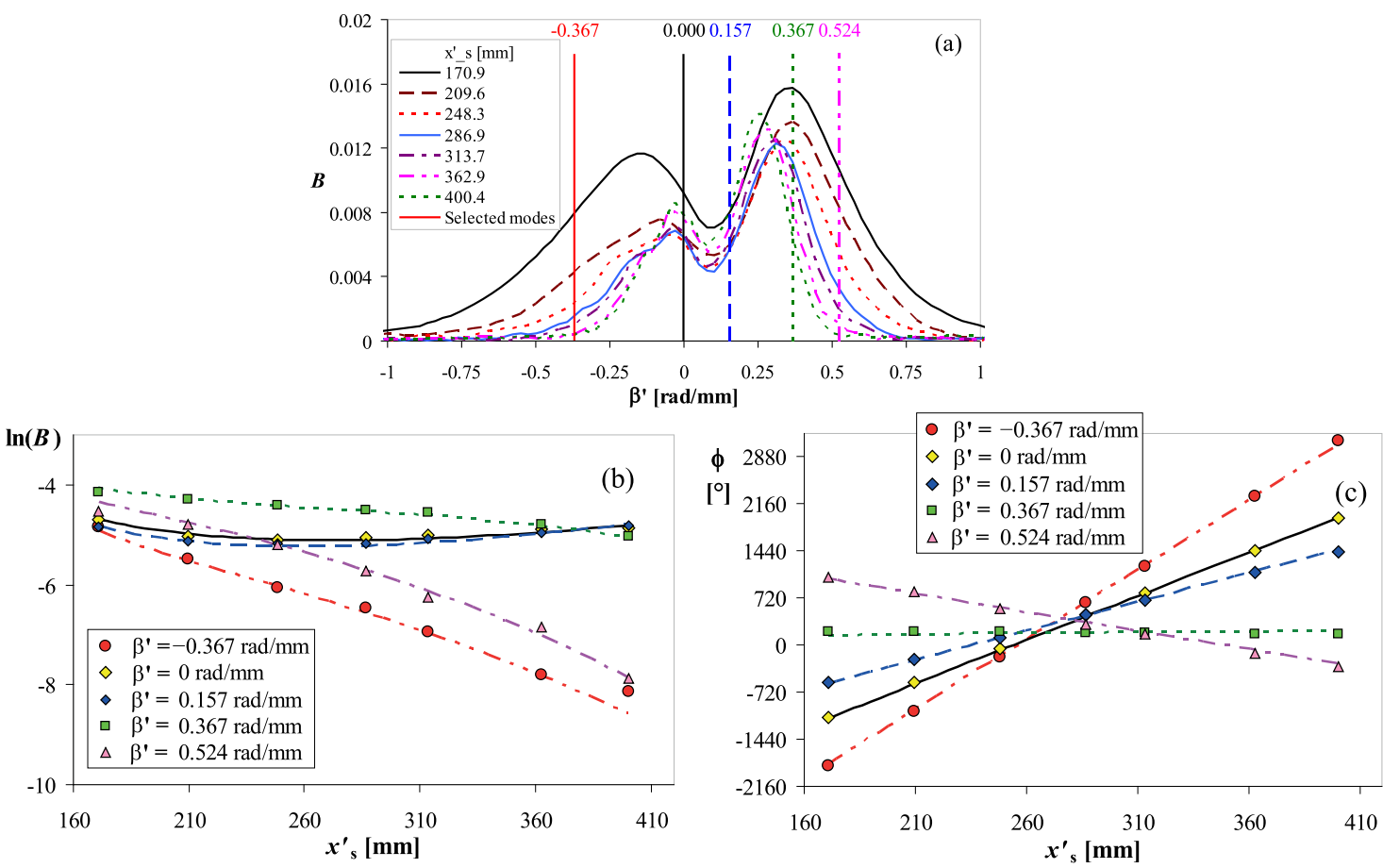

FIG. 14. Set of chordwise distributions of disturbance spectral amplitudes (b) and spectral phases (c) obtained experimentally (symbols) for $f=156 \mathrm{~Hz}$ in regime ZLF3(A6) from spectra shown in plot (a) at selected spanwise wavenumbers indicated by vertical lines. Corresponding theoretical distributions are shown with various lines.
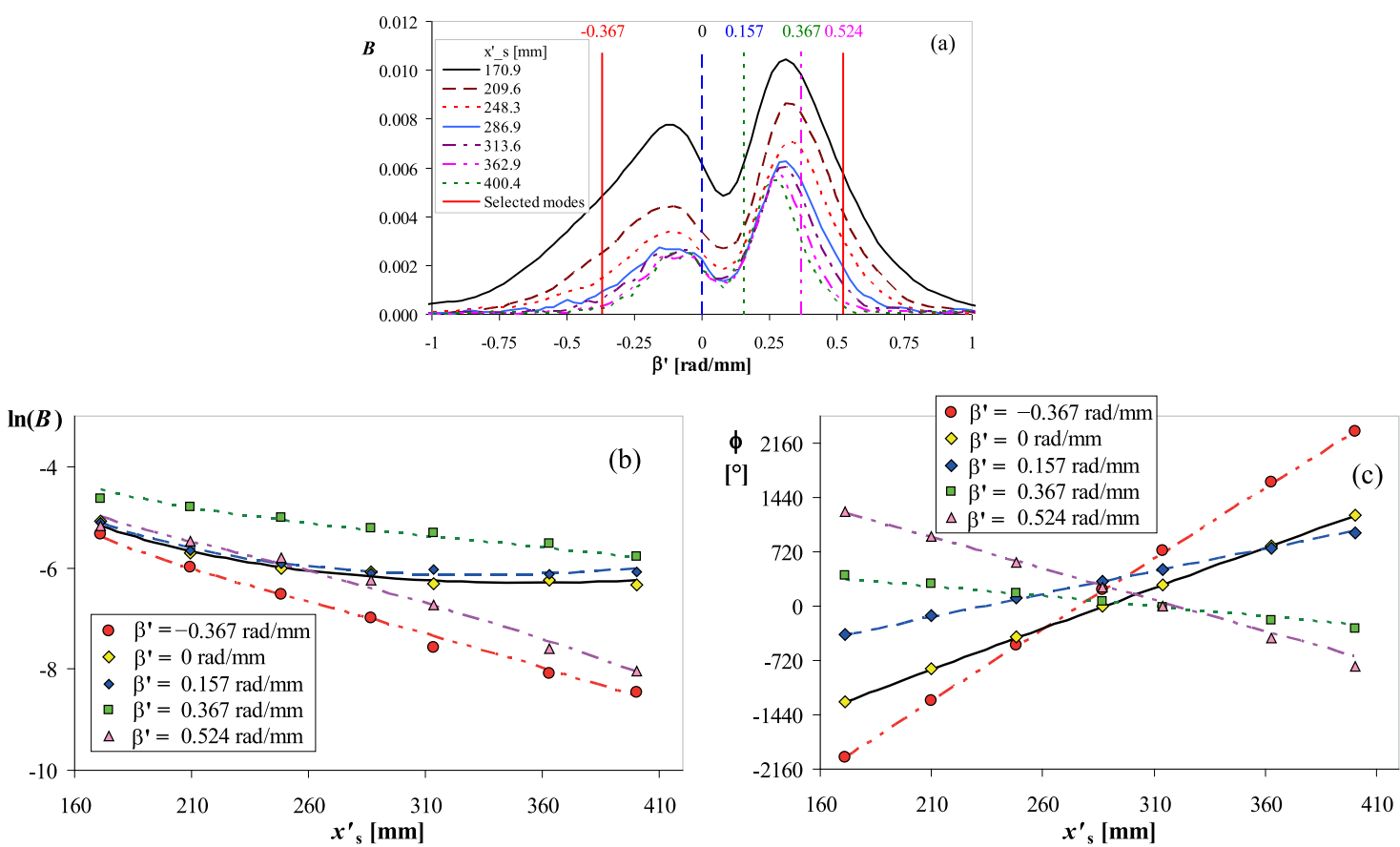

FIG. 15. Set of chordwise distributions of disturbance spectral amplitudes (b) and spectral phases (c) obtained experimentally (symbols) for $f=120 \mathrm{~Hz}$ in regime ZLF2(A6) from spectra shown in plot (a) at selected spanwise wavenumbers indicated by vertical lines. Corresponding theoretical distributions are shown with various lines. 

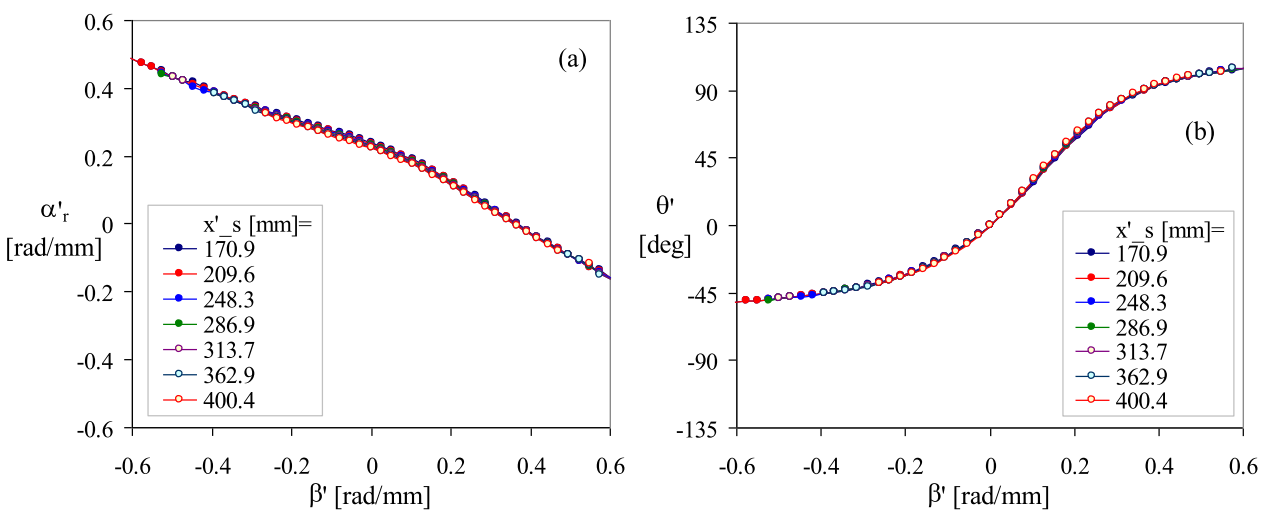

FIG. 16. Streamwise wavenumber $\alpha_{r}^{\prime}$ (a) and wave propagation angle $\theta^{\prime}$ (b) of normal TS-modes vs spanwise wavenumber $\beta^{\prime}$ measured in the $\left(x^{\prime}, z^{\prime}\right)$-coordinate system for frequency $f=156 \mathrm{~Hz}$ in regime ZLF3(A6) at various values of chordwise coordinate $x_{s}^{\prime}$.

\section{F. Measured characteristics of flow instability to 3D TS-modes}

Differentiation of the measured amplification curves like those shown in Figs. 13(c), 14(c), and 15(c) performed after their approximation by parabolas gave us the TS-wave streamwise wavenumbers $\alpha_{r}^{\prime}$ vs their spanwise wavenumbers $\beta^{\prime}$, i.e., the dispersion curves $\alpha_{r}^{\prime}\left(\beta^{\prime}\right)$. An example of a group of these curves obtained in regimes ZLF3(A6) $(f=156 \mathrm{~Hz})$ for various values of the streamwise coordinate is shown in Fig. 16(a). The corresponding TS-wave propagation angles $\theta^{\prime}=\tan ^{-1}\left(\beta^{\prime} / \alpha_{r}^{\prime}\right)$ are presented in Fig. 16(b).

The most physically important coordinate system for presentation of the instability characteristics on swept wings is the local $\left(x^{*}, z^{*}\right)$-system, which the $x^{*}$-axis is coupled with the potential-flow velocity vector taken at the boundary-layer edge. Three groups of dispersion curves $\alpha_{r}^{*}\left(\beta^{*}\right)$ and of the wave propagation angles $\theta^{*}$ vs the wavenumber $\beta^{*}$ measured at frequencies $f=194,156$, and $120 \mathrm{~Hz}$ [in regimes ZLF4(A6), ZLF3(A6), and ZLF2(A6)] are presented in Fig. 17(a) for various values of the streamwise coordinate. It is seen, in particular, that in the $\left(x^{*}, z^{*}\right)$-coordinate system, the dispersion curves [Fig. 17(a)] look rather symmetric with respect to the sign of the spanwise wavenumber in contrast to the $\left(x^{\prime}, z^{\prime}\right)$ coordinate system [cf. Fig. 16(a)] and in contrast to the $\alpha_{r}^{*}\left(\beta^{*}\right)$ distributions typical for the CF-instability modes (see, e.g., Ref. 1). The streamwise wavenumbers $\alpha_{r}^{*}$ increase with frequency for all values of the spanwise coordinate. Unlike to the CF-instability modes studied in Ref. 1, the TS-modes propagate in a broad range of relatively low absolute values of angles $\theta^{*}$ to the local potential-flow direction [Fig. 16(b)] between $\pm 75^{\circ}$ approximately, including zero propagation angles. At low frequencies, the absolute values of the TS-wave propagation angles $\theta^{*}$ increase faster with $\left|\beta^{*}\right|$ compared to higher frequencies [Fig. 16(b)].

Based on the streamwise wavenumbers, two kinds of phase speeds of the TS-instability modes have been obtained: (i) the phase speed $C_{K}=2 \pi f /|K|$ in the direction of wavevectors $K$ and (ii) the phase speed $C_{x}^{*}=2 \pi f / \alpha_{r}^{*}$ in the direction of the local potential flow velocity vector (i.e., in the $x^{*}$-direction). These two speeds are shown in Fig. 18(a) vs the spanwise wavenumber $\beta^{*}$ and in Fig. 18(b) vs the TS-wave propagation angle $\theta^{*}$, respectively, for the three most important studied frequencies [regimes ZLF4(A6), ZLF3(A6), and ZLF2(A6)] for all studied chordwise locations $x_{s}^{\prime}$. The two phase speeds are normalized by the absolute value $C_{e}$ of the local potential flow velocity vector measured at the boundary-layer edge. Note that these two phase speeds coincide with each other at $\beta^{*}=0$.

It is seen that the phase speed $C_{K}$ in the direction of wavevectors has its maximum value at $\beta^{*}=0$ and decays rather rapidly with $\left|\beta^{*}\right|$ but increases with frequency [Fig. 18(a)]. At large absolute values of the spanwise wavenumber $\beta^{*}$ (approximately at $\left|\beta^{*}\right|>0.3 \mathrm{rad} / \mathrm{mm}$ ), the phase speed $C_{K}$ is independent of the chordwise coordinate $x_{s}^{\prime}$, whereas at small values of $\left|\beta^{*}\right|$, it increases slightly with the
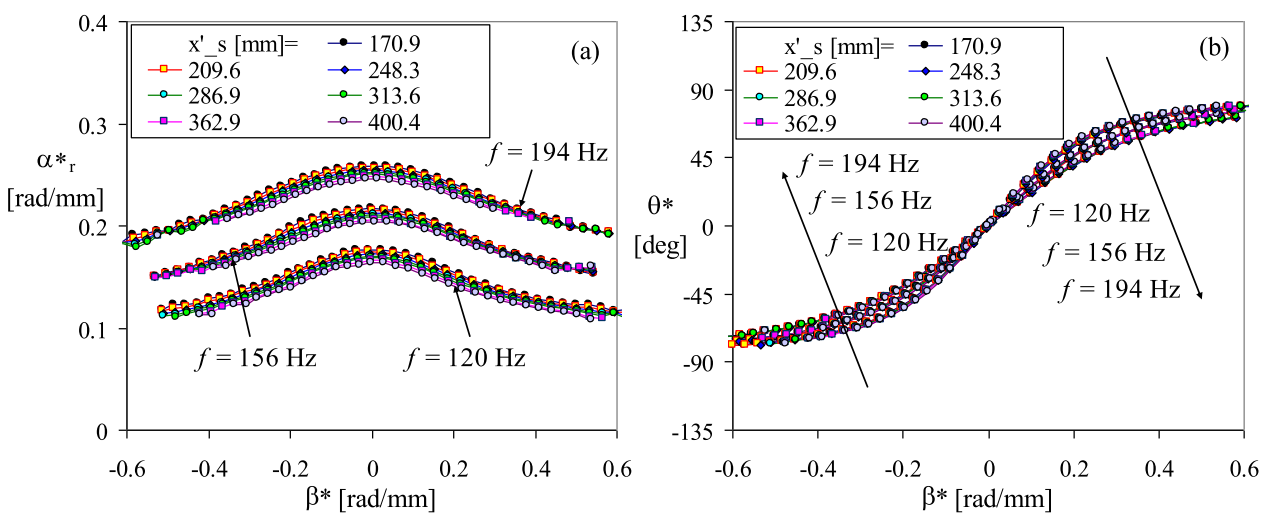

FIG. 17. Streamwise wavenumber $\alpha_{*}^{*}$ (a) and wave propagation angle $\theta^{*}$ (b) of normal TS-modes vs spanwise wavenumber $\beta^{*}$ measured in the local coordinate system for frequencies $f=194,156$, and $120 \mathrm{~Hz}$ in regimes ZLF4(A6), ZLF3(A6), and ZLF2(A6) at various values of chordwise coordinate $x_{s}^{\prime}$. 

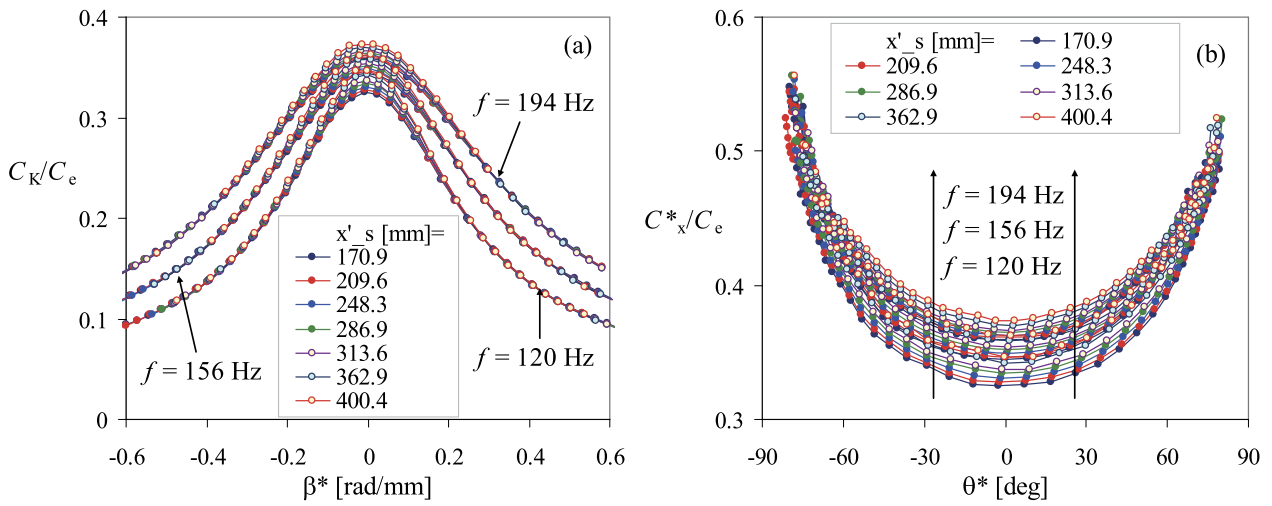

FIG. 18. Dimensionless phase velocities $C_{K}$ in the direction of wavevectors (a) and $C_{x}^{*}$ in the $x^{*}$-direction (b) measured in regimes ZLF4(A6), ZLF3(A6), and ZLF2(A6) for normal TS-modes with frequencies $f=194,156$, and $120 \mathrm{~Hz}$ at all studied chordwise locations $x_{s}^{\prime}$ vs spanwise wavenumber $\beta^{*}$ (a) and vs wave propagation angle $\theta^{*}(\mathrm{~b})$.

chordwise coordinate but remains below 0.4. Qualitatively, these distributions are rather similar to those inherent for the nonstationary CF-instability modes studied in Ref. 1.

The phase speed $C_{x}^{*}$ in the direction of the local potential flow velocity vector has the lowest values for quasi-2D modes (with $\left.\beta^{*}=0\right)$ and increases gradually with $\left|\beta^{*}\right|$ approaching values of about 0.6 [Fig. 18(b)]. These distributions are almost symmetric and differ very much from those inherent for the nonstationary CF-instability modes studied in Ref. 1. Indeed, in the latter case, the phase speeds $C_{x}^{*}$ of the CF-modes are completely asymmetric and even have second-order discontinuities at negative values of the spanwise wavenumber $\beta^{*}$ (and the wave propagation angles $\left.\theta^{*}\right)$; their frequency dependence is also extremely strong. It is also seen in Fig. 18(b) that the phase speed $C_{x}^{*}$ increases with the chordwise coordinate $x_{s}^{\prime}$ for every particular value of frequency and increases with frequency for every particular value of the chordwise coordinate.

Differentiation of the TS-mode amplification curves [like those shown in Figs. 13(b), 14(b), and 15(b)] gives us spatial increments $\left(-\alpha_{i}\right)$ of 3D TS-modes in the chordwise direction, which are shown in Figs. 19(a)-19(c) for frequencies $f=194,156$, and $120 \mathrm{~Hz}$, respectively [obtained in regimes ZLF4(A6), ZLF3(A6), and ZLF2(A6)]
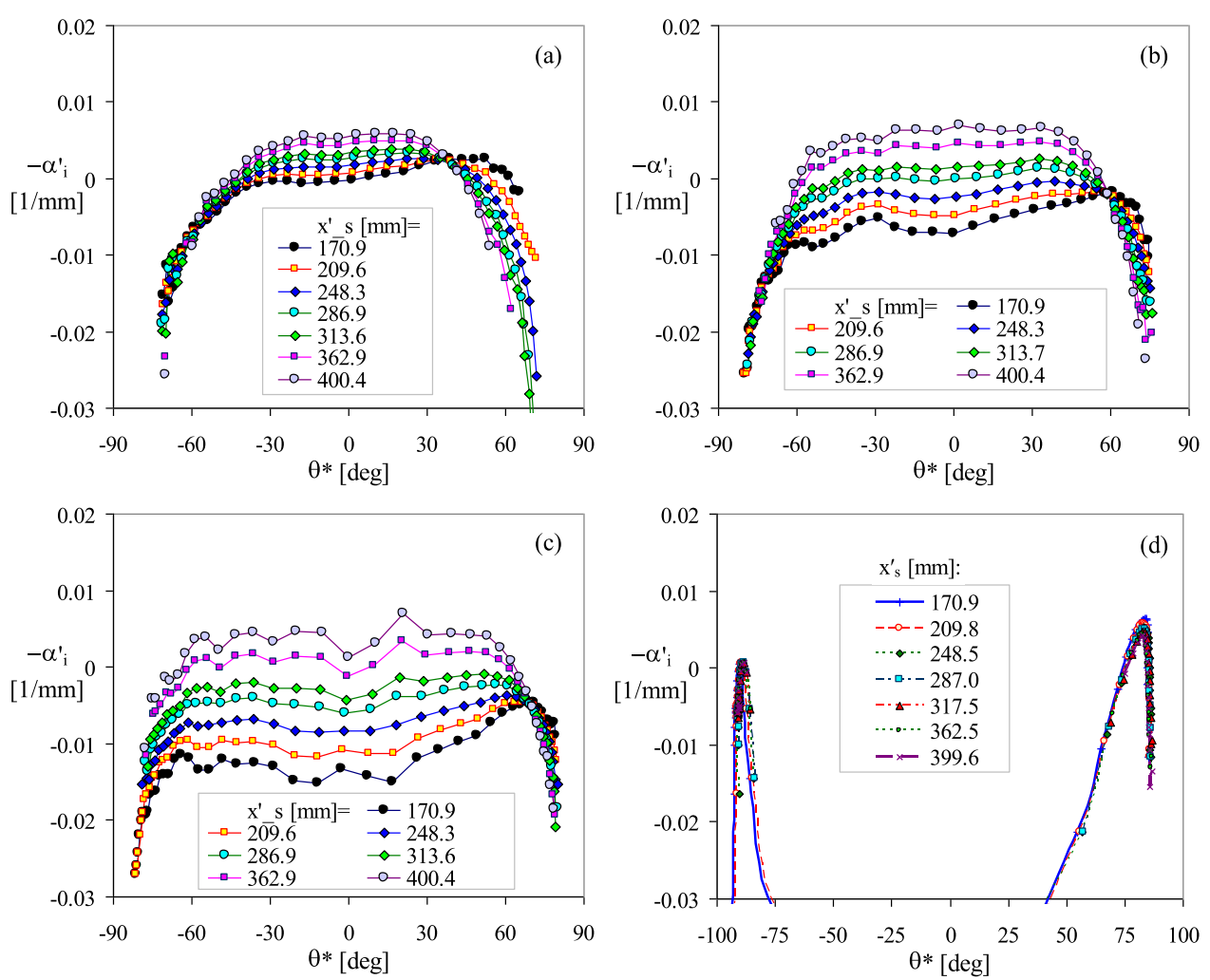

FIG. 19. Chordwise growth rates $\left(-\alpha_{i}\right)$ of normal TS-instability modes vs wave propagation angle $\theta^{*}$ (b) measured at various chordwise coordinates $x_{s}^{\prime}$ for frequencies $f=194 \mathrm{~Hz}(\mathrm{a}), 156 \mathrm{~Hz}(\mathrm{~b})$, and $120 \mathrm{~Hz}(\mathrm{c})$ in regimes ZLF4(A6), ZLF3(A6), and ZLF2(A6), respectively, and comparison with growth rates of normal CF-instability modes (d) obtained in experiments ${ }^{1}(f=40 \mathrm{~Hz})$ 

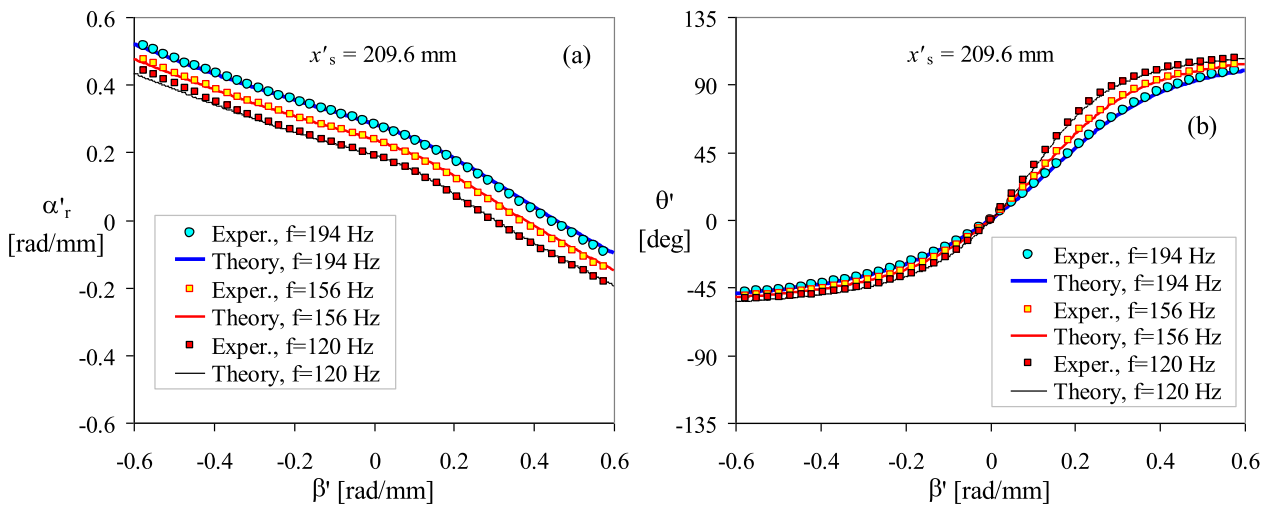

FIG. 20. Comparison of measured and calculated streamwise wavenumbers $\alpha_{r}^{\prime}$ (a) and wave propagation angles $\theta^{\prime}$ (b) of normal TS-modes vs spanwise wavenumber $\beta^{\prime}$ presented in the $\left(x^{\prime}, z^{\prime}\right)$-coordinate system for frequencies $f=194,156$, and $120 \mathrm{~Hz}$ at chordwise coordinate $x_{s}^{\prime}=209.6 \mathrm{~mm}$. vs the wave propagation angle $\theta^{*}$. The increments were obtained as derivatives of parabolic approximations of the experimental amplification curves taken in the logarithmic scale. The $-\alpha_{i}\left(\theta^{*}\right)$ distributions obtained for the TS-modes [Figs. 19(a)-19(c)] are compared with those measured in Ref. 1 for the nonstationary CF-modes [Fig. 19(d)].

First, it is seen that there is a large difference between shapes of the $-\alpha_{i}\left(\theta^{*}\right)$-distributions obtained for the TS- and CF-modes. The boundary layer is the most unstable or the least stable to those TS-waves which have relatively small absolute values of the wave propagation angles $\theta^{*}$ including the zero angle, while the CFinstability modes always attenuate in this range of $\theta^{*}$. In contrast to $2 \mathrm{D}$ boundary layers (the Blasius flow, for instance), the $-\alpha_{i}\left(\theta^{*}\right)$ distributions are typically nonsymmetric. In the beginning of the studied range of chordwise coordinate $x_{s}^{\prime}$, the most amplifying or the least attenuating TS-modes propagate at positive angles $\theta^{*}$ of about $50^{\circ}-70^{\circ}$ depending on frequency [Figs. 19(a)-19(c)]. These angles decrease with frequency. For every particular frequency, there is a point $\theta_{0}^{*}$ in the range of positive propagation angles, in which the increments are independent of the chordwise coordinate. Such point is not observed at negative $\theta^{*}$. The values of $\theta_{0}^{*}$ are, approximately, $40^{\circ}, 60^{\circ}$, and $70^{\circ}$ for frequencies $f=194,156$, and $120 \mathrm{~Hz}$, respectively [Figs. 19(a)-19(c)]. At propagation angles $\theta^{*}>\theta_{0}^{*}$, the amplification rates decrease with the chordwise coordinate, while at $\theta^{*}<\theta_{0}^{*}$, they increase. At large values of the chordwise coordinate, the $-\alpha_{i}\left(\theta^{*}\right)$-distributions become nearly symmetric although a small predominance of amplification of the modes with positive $\theta^{*}$ remains.

\section{G. Comparison of measured and calculated dispersion characteristics}

All measured dispersion characteristics are quantitatively compared below in detail with those calculated based on linear stability theory (LST). This comparison is presented for frequencies $f=194$, 156, and $120 \mathrm{~Hz}$.

Shown in Fig. 20 are the dispersion characteristics $\alpha_{r}^{\prime}\left(\beta^{\prime}\right)$ and $\theta^{\prime}\left(\beta^{\prime}\right)$ measured and calculated in the $\left(x^{\prime}, z^{\prime}\right)$-reference frame. It is seen, in particular, that there is not any symmetry of these distributions with respect to point $\beta^{\prime}=0$. The majority of the TS-modes propagates in the chordwise direction and has positive chordwise wavenumbers and absolute values of the wave propagation angle less than $90^{\circ}$. However, there are some TS-modes, having large positive spanwise wavenumbers $\beta^{\prime}$ (greater than about +0.2 or $+0.4 \mathrm{rad} / \mathrm{mm}$ for these particular frequencies), which propagate toward the airfoil leading edge, i.e., have negative values of $\beta^{\prime}$ and propagation angles $\theta^{\prime}>90^{\circ}$.

In the physically most relevant local $\left(x^{*}, z^{*}\right)$-coordinate system, used for presentation of the instability characteristics on swept wings, the same dispersion characteristics look rather symmetric
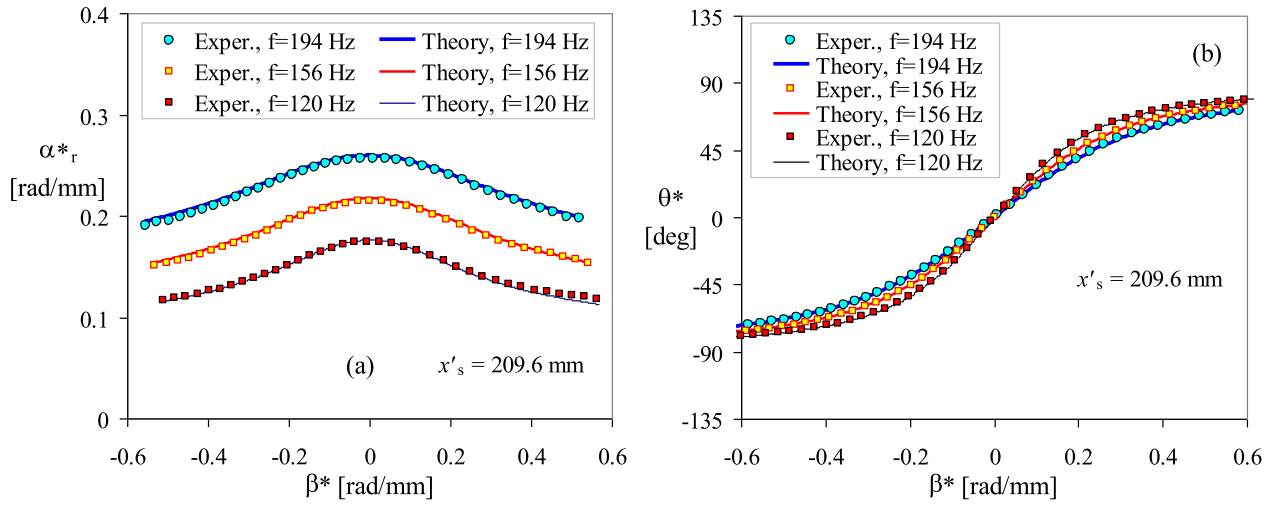

FIG. 21. Comparison of measured and calculated streamwise wavenumbers $\alpha_{*}^{*}$ (a) and wave propagation angles $\theta^{*}$ (b) of normal TS-modes vs spanwise wavenumber $\beta^{*}$ presented in the local coordinate system for frequencies $f=194,156$, and $120 \mathrm{~Hz}$ at chordwise coordinate $x_{s}^{\prime}=209.6 \mathrm{~mm}$. 

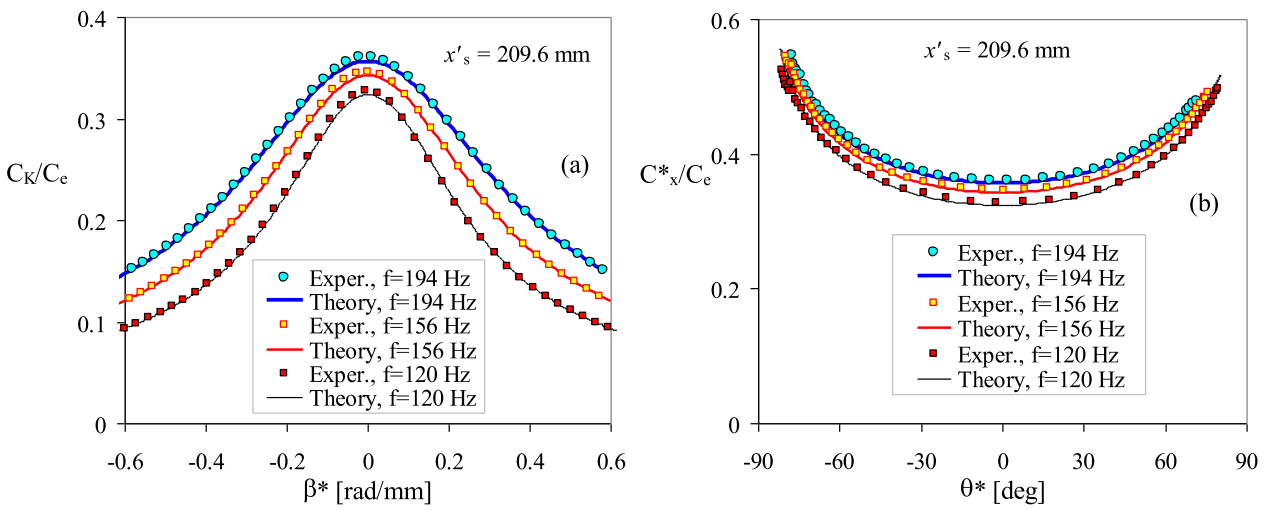

FIG. 22. Comparison of measured and calculated dimensionless phase velocities $C_{K}$, in the direction of wavevectors (a), and $C_{x}^{*}$, in $x^{*}$-direction (b) presented for normal TS-modes with frequencies $f=194,156$, and $120 \mathrm{~Hz}$ at chordwise coordinate $x_{s}^{\prime}=209.6 \mathrm{~mm}$ vs spanwise wavenumber $\beta^{*}$ (a) and vs wave propagation angle $\theta^{*}$ (b).
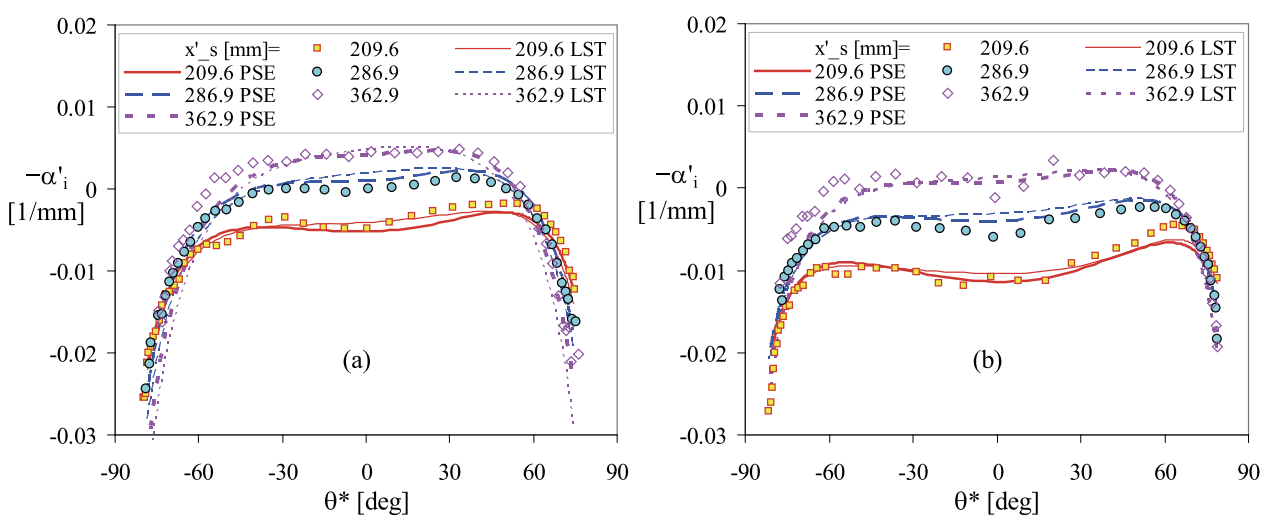

FIG. 23. Comparison of measured and calculated (LST- and PSE-approaches) chordwise growth rates $-\alpha_{i}$ of normal TS-instability modes vs wave propagation angle $\theta^{*}$ shown for three values of chordwise coordinate $x_{s}^{\prime}$ for frequencies $f=156$ (a) and 120 (b) $\mathrm{Hz}$.

(Fig. 21). Meanwhile, in contrast to 2D boundary layers (such as the Blasius flow), the symmetry is not exact. However, in this coordinate system, all TS-modes have positive streamwise wavenumbers $\alpha_{r}^{*}$ and propagate at angles $\theta^{*}$ having absolute values well below $90^{\circ}$.

The two TS-wave phase speeds (determined both in the wavevector direction and in the $x^{*}$ direction) are also almost symmetric but again not completely (Fig. 22). All measured stability characteristics shown in Figs. 20-22 demonstrate the applicability and accuracy of the numerical approaches used for analysis of the linear stability characteristics of the 3D boundary layer under study.

The comparison of the spatial amplification rates of 3D TSmodes estimated from the measured distributions with those calculated by the linear stability theory (LST) and by the PSE approach is illustrated in Fig. 23. Despite the accuracy of differentiation of the experimental amplification curves is always rather low (especially after performing a spanwise Fourier decomposition of the wavetrains excited by the spanwise-localized surface vibrator), the agreement between the measured and calculated chordwise growth rates is reasonably good for all values of (i) the chordwise coordinate, (ii) the spanwise wavenumber (i.e., the wave propagation angles), and (iii) the disturbance frequency. It is seen also that the PSE approach provides better agreement with the measurements, especially at low absolute values of the wave propagation angles. This indicates the importance of accounting for the base-flow nonparallelism and the surface curvature effects.

\section{CONCLUSIONS}

A detailed investigation of properties of boundary-layer disturbances evolving on a wing section modeling an infinite-span swept wing (with a sweep angle of $35^{\circ}$ ) has been performed in the framework of a combined experimental and theoretical study. The experiments are conducted at the angle of attack of $+1.5^{\circ}$ in the presence of a weak streamwise pressure gradient at completely controlled disturbance conditions excited by a special disturbance source. Due to this weak favorable pressure gradient, the perturbation field is dominated by the TS instability waves. In this case, the 3D cross-flow instability modes are practically not observed due to the absence of significant cross-flow in the presence of a nearly zero streamwise pressure gradient. The following most important results have been obtained.

All base-flow stability characteristics with respect to normal oblique TS-modes are obtained experimentally in a broad range of the spanwise-wavenumbers and frequencies and compared with those calculated (using the PSE and the LST) for the present experimental conditions. It is shown that the boundary-layer perturbations exited in the experiments have all properties corresponding to $3 \mathrm{D}$ TS-instability modes developing in accordance with the linear stability theory independent of the disturbance amplitude (in the studied amplitude range).

The experimental streamwise distributions of the normal instability mode amplitudes and phases are found to be in excellent 
agreement with those calculated based on the linear stability theory (using the PSE approach) for all studied oblique TS-modes. The corresponding estimated spatial amplification rates are found to agree with the calculated ones as well. A very good quantitative agreement is obtained also for amplitude and phase parts of the disturbance eigenfunctions and for all dispersion characteristics of the 3D TS-waves, including streamwise wavenumbers, wave propagation angles, and phase speeds as functions of the spanwise wavenumber and frequency. According to our knowledge, such agreement had never been achieved previously for TS-instability modes developing in $3 \mathrm{D}$ boundary layers, especially on real swept wings. Thus, we can conclude that the theoretical approaches used here are applicable to analysis of the stability problems of the swept-wing boundary layer with the TS-dominated transition scenario. The PSE approach is found to describe more accurately the stability characteristics observed experimentally.

The following most important properties of the 3D TS-waves developing in a swept-wing boundary layer are found.

The majority of the 3D TS-modes propagates in the chordwise direction although some of them, having large positive spanwise wavenumbers $\beta^{\prime}$, propagate toward the airfoil leading edge. This property is typical for the CF-instability modes of swept-wing boundary layers (see, e.g., Refs. 1 and 24) but has never being observed for 3D TS-waves developing in 2D boundary layers. The 3D TS-mode phase velocities, defined in the direction of the local potential flow, vary between 0.3 and 0.6 of $U_{e}$ and increase with the wave propagation angle similar to the $3 \mathrm{D}$ TS-modes studied in the 2D Blasius boundary layer.

The TS-mode eigenfunctions are qualitatively very similar to those found in a 2D Blasius boundary layer. ${ }^{13,14}$ Almost all of them have the amplitude maximum of the streamwise velocity disturbances located at $y / \delta_{1} \approx 0.77$ with $U / U_{e}=0.435$. Meanwhile, these eigenfunctions are significantly different from those typical for the CF-instability modes. ${ }^{1,3}$

In contrast to the CF-instability modes (studied in Ref. 1 on the same model but at $-5^{\circ}$ angle of attack; see also Ref. 33), the TS-modes are able to grow in a very broad range of the wavepropagation angle including quasi-2D ones (with $\beta^{\prime}=0$ ). However, the studied 3D boundary layer can never be unstable to the TSwaves propagating at very large angles $\theta^{*}$ close to $\pm 80^{\circ}-85^{\circ}$, while the CF-modes are the most amplified at these angles. ${ }^{1,24}$ Similar to the CF-instability modes, the most amplified TS-wave modes of the swept-wing boundary layer propagate upstream relative to the cross flow at local propagation angles $\theta^{*}$ between $25^{\circ}$ and $70^{\circ}$ approximately, depending on the disturbance frequency and the chordwise coordinate.

Thus, we can see that the 3D TS-instability modes developing in the studied swept-wing boundary layer have some properties similar to those known for 2D boundary layers, while other properties are completely different and remind those typical for the unsteady CFmodes developing in swept-wing flows.

One has to note in the end that the results of the present investigation provide a reliable foundation of subsequent study of the receptivity problems and, in particular, create a prerequisite for a detailed quantitative comparison of the measured and calculated characteristics of the swept-wing boundary-layer receptivity to surface nonuniformities and freestream vortices, which are the main subject of the RECEPT Project.

\section{ACKNOWLEDGMENTS}

This work was supported by the European Commission through FP7 project RECEPT (Grant Agreement No. ACPO-GA2010-265094) and carried out (partly) within the framework of the Program of Fundamental Scientific Research of the Russian state academies of sciences in 2013-2020 (Project No. AAAA-A17117030610128-8). The experimental study includes a significant contribution of P. H. Alfredsson, N. Tillmark, and A. Segalini at Department of Mechanics, KTH.

\section{REFERENCES}

${ }^{1}$ V. I. Borodulin, A. V. Ivanov, Y. Kachanov, D. Mischenko, R. Örlü, A. Hanifi, and S. Hein, "Experimental and theoretical study of swept-wing boundary-layer instabilities. Unsteady crossflow instability," Phys. Fluids 31, 064101 (2019).

${ }^{2}$ V. R. Gaponenko, A. V. Ivanov, Y. S. Kachanov, and J. D. Crouch, "Swept-wing boundary-layer receptivity to surface non-uniformities," J. Fluid Mech. 461, 93 (2002).

${ }^{3}$ W. S. Saric, H. L. Reed, and E. B. White, "Stability and transition of threedimensional boundary layers," Annu. Rev. Fluid Mech. 35, 413 (2003).

${ }^{4}$ P. Hall, M. R. Malik, and D. I. A. Poll, "On the stability of an infinite swept attachment line boundary layer,” Proc. R. Soc. London, Ser. A 395, 229 (1984).

${ }^{5}$ W. E. Gray, "The effect of wing sweep on laminar flow," RAE TM Aero No. 227, 1952.

${ }^{6} \mathrm{U}$. Dallmann and H. Bieler, "Analysis and simplified prediction of primary instability of three-dimensional boundary-layer flows," in 19th AIAA, Fluid Dynamics, Plasma Dynamics, and Lasers Conference (American Institute of Aeronautics and Astronautics, 1987).

${ }^{7}$ M. V. Ustinov, "Laminar-turbulent transition in boundary layers (review). Part. 1: Main types of laminar-turbulent transition in a swept-wing boundary layer," TsAGI Sci. J. 44, 1 (2013).

${ }^{8}$ A. Dovgal, V. Kozlov, and A. Michalke, "Laminar boundary layer separation: Instability and associated phenomena,” Prog. Aerosp. Sci. 30, 61 (1994).

${ }^{9}$ M. Gaster, I. Grant, and J. T. Stuart, "An experimental investigation of the formation and development of a wave packet in a laminar boundary layer," Proc. R. Soc. London, Ser. A 347, 253 (1975).

${ }^{10}$ V. M. Gilyov, Y. S. Kachanov, and V. V. Kozlov, "Development of a spatial wave packet in a boundary layer," Izv. Sib. Otd. Akad. Nauk SSSR, Ser. Tekh. Nauk 13(3), 27 (1983) (in Russian).

${ }^{11}$ Y. S. Kachanov, "Development of spatial wave packets in boundary layer," in Laminar-Turbulent Transition, edited by V. V. Kozlov (Springer, Berlin Heidelberg, 1985), pp. 115-123.

${ }^{12}$ Y. S. Kachanov and T. G. Obolentseva, "Development of three-dimensional disturbances in the Blasius boundary layer. 1. Wave-trains," Thermophys. Aeromech. 3(3), 225 (1996).

${ }^{13}$ Y. S. Kachanov and T. G. Obolentseva, "Development of three-dimensional disturbances in the Blasius boundary layer. 2. Stability characteristics," Thermophys. Aeromech. 4(4), 373 (1996).

${ }^{14}$ Y. S. Kachanov and T. G. Obolentseva, "Development of three-dimensional disturbances in the Blasius boundary layer. 3. Nonparallelism effects," Thermophys. Aeromech. 5(3), 331 (1998).

${ }^{15}$ W. Würz, S. Herr, A. Wörner, U. Rist, S. Wagner, and Y. S. Kachanov, "Threedimensional acoustic-roughness receptivity of a boundary layer on an airfoil: Experiment and direct numerical simulations," J. Fluid Mech. 478, 135-163 (2003).

${ }^{16}$ Y. S. Kachanov and D. B. Koptsev, "Three-dimensional stability of self-similar boundary layer with a negative Hartree parameter. 1. Wave-trains," Thermophys. Aeromech. 6(4), 443 (1999).

${ }^{17}$ Y. S. Kachanov, D. B. Koptsev, and B. V. Smorodsky, "Three-dimensional stability of self-similar boundary layer with a negative Hartree parameter. 2. Characteristics of stability," Thermophys. Aeromech. 7(3), 341 (2000).

${ }^{18}$ F. P. Bertolotti, "Linear and nonlinear stability of boundary layers with streamwise varying properties," Ph.D. thesis, The Ohio State University, 1991. 
${ }^{19}$ D. Romano, P. Alfredsson, A. Hanif, R. Örlü, N. Tillmark, V. Borodulin, A. Ivanov, Y. Kachanov, and M. Minervino, "Design and tests of wind-tunnel sidewalls for receptivity experiments on a swept wing," in Mechanical and Aerospace Engineering IV, Applied Mechanics and Materials (Trans Tech Publications, 2013), Vol. 390, pp. 96-102.

${ }^{20}$ A. Hanif, S. Hein, D. Romano, M. Minervino, W. Würz, V. Borodulin, A. Ivanov, and Y. Kachanov, "Numerical and experimental realization of an infinite-swept-wing boundary-layer flow in a wind tunnel," in Proceedings of 17th International Conference on the Methods of Aerophysical Research, edited by V. Fomin (ITAM SB RAS, Novosibirsk, 2014).

${ }^{21}$ V. Borodulin, A. Ivanov, and Y. Kachanov, "Numerical and experimental realization of an infinite-swept-wing boundary-layer flow in a wind tunnel," in Proceedings of 17th International Conference on the Methods of Aerophysical Research, edited by V. Fomin (ITAM SB RAS, Novosibirsk, 2014).

${ }^{22}$ Y. Kachanov, O. Tararykin, and A. Fedorov, "Investigation of stability of boundary layer on a model of a swept wing to stationary disturbances," Izv. Sib. Otd. Akad. Nauk SSSR, Ser. Tekh. Nauk 5, 11 (1990) (in Russian).

${ }^{23}$ A. V. Ivanov, Y. S. Kachanov, and D. A. Mischenko, "Generation of nonstationary Görtler vortices by localized surface nonuniformities. Receptivity coefficients," Thermophys. Aeromech. 19, 523 (2012).

${ }^{24}$ V. R. Gaponenko, A. V. Ivanov, and Y. S. Kachanov, "Experimental study of cross-flow instability of a swept-wing boundary layer with respect to traveling waves," in Laminar-Turbulent Transition, edited by R. Kobayashi (Springer, Berlin Heidelberg, 1995), pp. 373-380.

${ }^{25}$ V. I. Borodulin, A. V. Ivanov, Y. S. Kachanov, R. Örlü, A. Hanifi, and S. Hein, "Characteristics of 3D instability of a 35-degree swept wing to CF and TS modes. Experiment and theory," AIP Conf. Proc. 1770, 030054 (2016).
${ }^{26}$ A. V. Boiko, A. V. Ivanov, Y. S. Kachanov, and D. A. Mischenko, "Steady and unsteady Görtler boundary-layer instability on concave wall," Eur. J. Mech., B: Fluids 29, 61 (2010).

${ }^{27}$ V. I. Borodulin, A. V. Ivanov, Y. S. Kachanov, and D. A. Mischenko, "Systematic study of distributed excitation of unsteady Görtler modes by freestream vortices," Eur. J. Mech., B: Fluids 68, 167 (2018).

${ }^{28}$ A. Hanifi, D. Henningson, S. Hein, F. Bertolotti, and M. Simen, "Linear nonlocal instability analysis-The linear NOLOT code," Technical Report No. FFA TN 54, 1994.

${ }^{29}$ S. Hein, F. Bertolotti, M. Simen, A. Hanifi, and D. Henningson, "Linear nonlocal instability analysis-The linear NOLOT code," Technical Report No. DLR-IB 22394 A43, 1994

${ }^{30}$ A. V. Ivanov, Y. S. Kachanov, and T. G. Obolentseva, "Experimental study of Blasius boundary layer receptivity to localized surface vibrations," Thermophys. Aeromech. 6(2), 179 (1999)

${ }^{31}$ Y. S. Kachanov and A. Michalke, "Three-dimensional instability of flat-plate boundary layers: Theory and experiment," Eur. J. Mech., B: Fluids 13, 401 (1994).

${ }^{32}$ J. D. Crouch, V. R. Gaponenko, A. V. Ivanov, and Y. S. Kachanov, "Receptivity of swept-wing boundary layer to microscopic surface nonuniformities. Theory and experiment," in Vth International Workshop on the Stability of Homogeneous and Heterogeneous Fluids Flows, Part III (NGASU, Novosibirsk, 1998), pp. $122-130$ (in Russian).

${ }^{33}$ V. R. Gaponenko, A. V. Ivanov, and Y. S. Kachanov, "Experimental study of a swept-wing boundary-layer stability with respect to unsteady disturbances," Thermophys. Aeromech. 2(4), 287 (1995). 